* Medienverbund anno 1930: Emil und die Detektive

\author{
* Medienverhalten und Medienkritik \\ jugendlicher Musikexperten \\ * „Wag the Dog"“ und Medienkritik
}

\section{Medienverbund anno 1930: Emil und die Detektive. Erich Kästner und der Markt}

\section{KATHARINA HÄFELE}

Wenn heute ein neues Buch auf den Markt kommt, wird meist gleich geplant, welche Merchandising-Produkte begleitend herausgebracht werden können. Spätestens, wenn die erste Auflage vergriffen ist, wird eine weitere Vermarktung des Stoffes in anderen Medien erwogen. PC-Spiele, Filme, Hörkassetten, ein Fan-Club ist über den Verlag und die eigene Homepage anzusteuern und fördert das Gespräch über den Stoff. Natürlich steht dahinter ein kommerzielles Interesse, aber auch die Nutzer haben einen Mehrwert. Schon Erich Kästner nutzte 1929 die Möglichkeit der Mehrfachverwertung bei der Vermarktung seines ersten Romans Emil und die Detektive. Damit war er ungewöhnlich fortschrittlich. Ich werde versuchen, Parallelen zwischen der frühen Vermarktung von Emil und die Detektive und heutiger medienkonvergenter Vermarktung zu ziehen und am Ende des Aufsatzes einige didaktische Anmerkungen machen. Damit soll ein neuer Blick auf den Autor und sein inzwischen zum Klassiker avanciertes Jugendbuch geworfen werden.

\section{Medienverbund und Medien- konvergenz}

Die Terminologie im Hinblick auf die verschiedenen medialen Verflechtungen erscheint häufig unübersichtlich. Am Beispiel von Emil und die Detektive soll zunächst versucht werden, Begrifflichkeiten von einander abzugrenzen und sie für den didaktischen Gebrauch vereinfachend zu bestimmen. Zunächst sollen Medienverbund und Medienkonvergenz unterschieden werden:

Der Begriff „Medienverbund“ meint, dass ein und derselbe Inhalt in unterschiedlichen Medien vorkommt. Dabei behält trotzdem jedes Medium seine individuelle Funktion. Wichtig sind vor allem auch die ökonomischen Verflechtungen von Medien. Wenn Emil und die Detektive also als Roman, als Film und als Gesellschaftsspiel auf den Markt kommt, haben wir solch einen Medienverbund. Die Medien werden distinkt betrachtet und vermarktet. Die Funktion der einzelnen Angebote bleibt klar unterschieden." (Hasebrink/Mikos/Prommer 2004, 11) Diese Verflechtung tritt jedoch nicht quasi naturwüchsig auf, sondern ist gerade in Kindermedien als Verbund geplant (vgl. Hurrelmann/Becker 2003, 5).

Der Begriff „Medienkonvergenz“ dagegen wird hier in Deutschland vor allem im Zusammenhang mit medienübergreifendem Verhalten verwendet (vgl. Wagner/ Theunert 2006), bei dem sich ein Mehrwert der größeren Wahlmöglichkeit für den Rezipienten sowie ein Mehrwert für den Autor oder den Vertreiber ergeben (vgl. MarciBoehncke 2007, 134). Insbesondere bei Medienangeboten für Kinder kommt es zur Mehrfachverwertung. Als erfolgreiches aktuelles Beispiel kann die Vermarktung von Harry Potter und Die wilden Fußballkerle genannt werden (ebd., 131).

\section{Die Vermarktung von Emil und die Detektive}

Der Roman Emil und die Detektive erschien erstmals 1929 und war Erich Kästners erster Roman. Er wurde ein großer Erfolg und zählt heute zu den Kinderbuchklassikern. Rund 2,2 Millionen Exemplare sind verkauft worden und der Roman wurde in 57 Sprachen übersetzt (Pressemappe Erich Kästner, 33). An den Erfolg anknüpfend gab es in den 1930er Jahren eine Bühnenfassung und einen Film. Heute ist der Stoff in zahlreiche Medien umgesetzt (vgl. Tabelle auf der nächsten Seite).

Für den großen Erfolg kann neben der Qualität des Stoffes auch die Art der Verbundvermarktung vermutet werden. Der Roman war das Ausgangsmedium, die übrigen Umsetzungen profitierten von der Popularität des Stoffes und dem Wunsch der Rezipienten, den Protagonisten auch in anderen Medien zu begegnen.

Vermarktung durch die Kinderzeitung von Klaus und Kläre

Eine Möglichkeit der Vermarktung seines Romans fand Kästner mit der Kinderzeitung von Klaus und Kläre. Diese Kinderzeitung war Teil des Familienblattes „Beyers für alle“, für das Kästner während seiner Berliner Zeit neben der Neuen Leipziger Zeitung schrieb. 
Medienverbund bei „Emil und die Detektive“

\begin{tabular}{|c|c|}
\hline Vor 1945 & Nach 1945 \\
\hline $\begin{array}{l}\text { - } \text { Roman (1929) } \\
\text { - } \text { Film (1931) } \\
\text { - } \text { Filmspiel } \\
\text { - Gesellschaftsspiel (Jos. Scholz, 1931) } \\
\text { - Bühnenfassung (1930) } \\
\text { - Nachfolgeroman „Emil und die } \\
\text { drei Zwillinge“ (1933) } \\
\text { - Bühnenfassung „Emil und die drei Zwillinge“ }\end{array}$ & $\begin{array}{ll}\text { - } & \text { 2. Bühnenfassung (1998) } \\
\text { - } & \text { Musical (2000) } \\
\text { - } & \text { Hörspiel: CD, Kassette, LP } \\
\text { - } & \text { Lesung (CD) } \\
\text { - } & \text { Hörspiel „Emil und die drei Zwillinge“: } \\
& \text { CD, Kassette, LP } \\
\text { - } & \text { Gesellschaftsspiel (Schmidt Spiele) } \\
\text { - } & \text { Weitere Filmversionen (DVD, VHS) }\end{array}$ \\
\hline
\end{tabular}

Quellen: Pressemappe Erich Kästner; www.amazon.de; Harbusch (1999)

Kästner erhielt zahlreiche Briefe und Anrufe von Kindern, die wissen wollten, ob die Geschichte von Emil Tischbein wahr sei (vgl. im Folgenden Harbusch 1999, 9). Diese Rückmeldung der Kinder nutzte Kästner in Form von abgedruckten Leserbriefen strategisch, um für seinen Roman zu werben. „Diese Kinderbeilage“ - so Harbusch (ebd., 10) - ,ist das Musterbeispiel eines erfolgreichen Werbefeldzuges und dafür, wie man Kinder als Käufer, Leser und Autoren mobilisiert." Immer wurden die Leser der Kinderzeitung miteinbezogen, z. B. durch Umfragen und Briefaktionen. Diese multimediale Art, Medienkonsumenten an sich zu binden, wird heute mit dem Begriff Medienkonvergenz. beschrieben. Insbesondere die Kommunikation zwischen Autor und/oder Verlag und Rezipienten, die die Kinderzeitung über Leserbriefe ermöglichte, stellte eine frühe Form der analogen Interaktivität dar. Sonstige Push-Angebote (Medien geben eine Information an die Rezipienten) wurden zu wechselseitig funktionierenden push and pull-Medien erweitert. Kästner konnte den Markt beeinflussen, aber nutzte auch die Rückkoppelung mit seinen Rezipienten, um die Akzeptanz seines Romans zu erheben - also qualitative Marktforschung zu betreiben.

Vergleichbar ist dies der Interaktivität, die heute wieder über Internetseiten ermöglicht wird, um die Konsumenten zu binden und um auf die Interessen der Konsumenten angemessen reagieren zu können. Auch für Emil und die Detektive wurde bereits in analogen Mediensystemen auf diese Art und Weise geworben. Es war die Idee der Verlegerin Edith Jacobsohn, ,die Begeisterung der Kinder zu Werbezwecken zu nutzen“ (ebd., 11). Auch den Brief eines begeisterten Jungen, der in Berlin Straßen abgelaufen war, die in Emil und die Detektive vorkamen, wollte Jacobsohn vervielfältigen lassen und als Reklame benutzen. Das tat sie auch, nicht nur den Brief des Jungen, sondern auch den Brief einer Klasse benutzte sie für einen Wer- beprospekt. In diesem Werbeprospekt wurde wiederum dazu aufgerufen, Kästner doch Briefe zu schicken. Die Kommunikation, die zwischen Leser und Autor zustande kam, kann auch mit dem Erfolg von Film und Theater sowie den Erfolg weiterer Kästnerbücher in Verbindung gebracht werden. Unter dem Namen „Kästner“ konnten sich die Kinder nun etwas vorstellen. Die Fanpost benutzte Kästner für einen Artikel mit der Überschrift „Mein ,kleines’ Publikum“, den er verschiedenen Zeitungen gab (vgl. Harbusch 1999, 12). Zusammenfassend lässt sich sagen: Die Leserbriefaktion war nicht nur deshalb wirksam, weil die Kinder mit einbezogen wurden, sondern auch, weil die Kinder selbst durch ihre positive Kritik weitere Leser warben, denn ihre Kritik war für Kinder sicher glaubwürdiger als die Kritik eines Erwachsenen. Des Weiteren konnten die Briefe benutzt werden, um Vorschläge und Wünsche der Kinder zu erfahren und darauf zu reagieren: Der Vorschlag, aus Emil und die Detektive einen Film zu machen, kam, glaubt man der Darstellung Kästners, unter anderem von den jungen Lesern und auch eine Fortsetzung wurde gewünscht (ebd.).

\section{Der Film von 1931}

Der Vorschlag zu einem Film entsprach sowohl dem Wunsch der Leser als auch der Kritiker von Emil und die Detektive. Kästner, der sich selbst sehr für den Film interessierte und später im Natinalsozialismus dort auch auf umstrittene Art und Weise unter dem Pseudonym „Berthold Bürger“ als Drehbuchautor des NS-Renommier-Kostümfilms „Münchhausen“ eine Einnahmequelle fand (vgl. Hofmann 1995, 162), stimmte der Idee einer Verfilmung zu. Die Rechte an einem Film von Emil und die Detektive erwarb die Ufa 1930 (vgl. Lutz-Kopp 1993, 22). Regie führte Gerhard Lamprecht, am Drehbuch hatte Kästner selbst mitgearbeitet (vgl. Schmid 2000, 24). Wie bereits zuvor bei der Werbung für den Roman, wurde auch der 
Film auf strategische Weise vermarktet. 1260 Kinder wurden gecastet und die Zeitungen berichteten darüber. Auch bei den Dreharbeiten war die Presse dabei und machte Interviews mit den jungen Schauspielern. AuBerdem schrieb Kästner vor der Premiere einen Artikel darüber, wie er in der realen Welt seinen eigenen Figuren begegnete: er geriet aus Versehen in die Filmarbeiten von Emil und die Detektive (vgl. Harbusch 1999, 14). Da Kästner als Journalist bekannt war, bot sich ihm diese Möglichkeit, in der Zeitung für seinen eigenen Film zu werben. Der Film wurde, ähnlich wie der Roman, international erfolgreich (vgl. Tornow 1998, 35). 1935 wurde Emil und die Detektive auch in Großbritannien verfilmt (Harbusch 1999, 40). Tornow (1998, 40f.) listet insgesamt sechs Verfilmungen auf. Dazu kommt noch die Neuverfilmung von 2001 „Das neue Filmspiel".

Eine besondere Vermarktungsstrategie war der Einstieg in das Medium „Spiel“, das schon im Umfeld des ersten Filmes von der Ufa heraus gebracht wurde: Das neue Filmspiel (vgl. im Folgenden Harbusch 1999). Die Spielfiguren müssen von den Spielern selbst ausgeschnitten werden: Es sind Fotos der Schauspieler aus dem Film, was die Identifikation der Spieler mit den Figuren erleichtert. Überhaupt führt das Mitspielen zu einer Teilnahme des Spielers an der spannenden Verbrecherjagd und lässt idealiter auch Anschlusshandlungen über das Gesellschaftsspiel hinaus zu: Kinder etwa könnten die Handlung im freien Rollenspiel nachstellen und selbst zu „Schauspielern“ werden. Neben der Spielanleitung steht der Zusatz: „Ganz richtig werdet ihr diese Gründe natürlich nur verstehen, wenn Ihr Euch den Film Emil und die Detektive angesehen habt." Damit wird ganz offensichtlich für den Film geworben: Wer den Hintergrund des Spielaufbaues verstehen möchte, muss den Film ansehen. Auf der Rückseite lobt ein Text den Film und gibt Auskunft über den Inhalt. Erwachsene sollen sich auch angesprochen fühlen, was schon auf der Vorderseite angedeutet wird mit „, für alle jungen Menschen zwischen 70 und 6": ,Dieser Film macht in Ihnen alles wieder lebendig, was Sie an Sonnigem, Sorglosem, Lachendem aus Ihrer Jugendzeit in Ihrem Herzen bewahrt haben." So versucht dieses Werbespiel, die Kinder über die Erwachsenen zu erreichen. Das Entscheidende folgt nach dem Werbetext: Die Vorankündigung der Spielzeiten des Filmes inklusive Ortsangabe und Eintrittspreise. Neben dem Film wird gleichzeitig auch auf das Buch, seinen Vertrieb und Preis verwiesen.

Auch der Mainzer Spieleverlag Jos. Scholz bringt zu Weihnachten 1931 ein Gesell- schaftsspiel Emil und die Detektive heraus (vgl. Harbusch 1999, 16). Das Filmjournal schlägt Theaterbesitzern im zeitgemäßen Jargon vor, mit Buchhandlungen und Spielzeugläden eine „Gemeinschafts-Schaufensterpropaganda" zu machen (ebd.). So verweist jedes Medium auf zwei andere.

\section{Fortsetzung in Emil und die drei Zwillinge}

In dem Fortsetzungsroman Kästners Emil und die drei Zwillinge von 1934 wird ebenfalls auf andere Medien verwiesen. Im Vorwort, das aus zwei Teilen besteht (ein Vorwort für „Laien“ und ein Vorwort für „Fachleute"), verweist Kästner auf seinen ersten Roman. Kästner lässt die Figuren aus Emil und die Detektive Zuschauer ihres eigenen Films werden - Emil und die Detektive wird in Emil und die drei Zwillinge - genauso wie in der Realität - verfilmt. Kästner machte so nicht nur ,auf äußerst intelligente Art [...] Werbung“" (Schmid 2000, 25) für seinen ersten Roman und dessen Verfilmung: Roman und Film werden vielmehr intertextuell verbunden (vgl. Marci-Boehncke 2007, 23).

Es deutet einiges daraufhin, dass Kästner den Roman Emil und die drei Zwillinge aus Geldnot veröffentlichte, denn nach der Machtübernahme waren Kästners Werke nach und nach verboten worden. Schmid (2000, 25) bezeichnet diese Vermarktungsstrategie als „Überlebensstrategie“ Kästners, weil er in Deutschland seit 1933 nicht mehr publizieren durfte. Emil und die drei Zwillinge musste zwar in der Schweiz veröffentlicht werden, aber man konnte den Roman auch in Deutschland kaufen. Trotzdem war Kästner ökonomisch unter Druck. Thomas Anz (1998, 780) betont daher im Hinblick auf Kästners Praktik, Stoffe mehrfach zu verwerte: „In der Zeit des Nationalsozialismus wurde [die Mehrfachverwertung, $\mathrm{KH}$ ] zu einer ökonomischen Überlebensfrage." Kästner versuchte, auf dem Erfolg, den er mit Emil und die Detektive hatte, aufzubauen. Auch aus „Emil und die drei Zwillinge“ wird eine Bühnenfassung gemacht (vgl. Harbusch 1999, 19). Trotzdem blieb Emil und die drei Zwillinge im Vergleich zu Kästners anderen Kinderbüchern relativ erfolglos (vgl. Lutz-Kopp 1993, 21).

\section{Weitere Medienformen nach 1945}

1947 erschien Emil und die Detektive als Rotationsroman in der Reihe der Williams Jugendbefte (vgl. Haack 2007, 37f.). Kästners Popularität setzte sich nach dem Krieg scheinbar ungebrochen fort. Mit der Weiterentwicklung der Medienlandschaft wurde dieser erfolgreiche Stoff auch in andere mediale Formen geführt. Ein Remake der ersten Verfilmung wurde 1954 gedreht, eine Zeit, in der 
Kästner ein beeindruckendes Comeback erlebt (vgl. Tornow 1998, 120). Es folgten auch Verfilmungen von Emil und die Detektive in Japan, Brasilien, USA, Ungarn und Argentinien (vgl. Habusch 1999, 18). Von Emil und die Detektive gibt es auditive Fassung, vergleichbar heutigen Hörbüchern als MC und $\mathrm{CD}$, früher selbstverständlich als LP (Pressemappe Erich Kästner, 30). Auch Emil und die drei Zwillinge wurde vertont, immer noch gibt es auch ein Gesellschaftsspiel Emil und die Detektive aus dem Hause Schmidt Spiele. 2001 entstand eine deutsche Neuverfilmung von Emil und die Detektive von Regisseurin Franziska Buch. Es gibt mehrere „Emil“-Musicals (vgl. Harbusch 1999, 18) und „literarische Berlin-Führer“" gehen mit Interessierten auf Spurensuche, als Buch oder leibhaftig (ebd.).

Obwohl Emil und die Detektive inzwischen als Klassiker gilt, der eine gezielte Vermarktung nicht mehr nötig hat, funktioniert der Verweis des einen Mediums auf das andere heute noch genauso gut wie im vorigen Jahrhundert.

\section{Didaktische Überlegungen}

\section{Medienkompetenz in der Schule}

Die immer rasanteren Entwicklungen im Bereich Medien im 20. und 21. Jahrhundert stellen auch die Schulen vor neue Aufgaben. Medienkompetenz darf nicht mehr nur den Umgang mit Printmedien betreffen (vgl. Gast/ Marci-Boehncke 1996; Hurrelmann/Becker 2003, 5). Bei den für Kinder bestimmten Medien handelt es sich dabei immer öfter um Medienverbünde. Hurrelmann/Becker (2003, 11) sprechen von einer „Integration schriftsprachlicher Fähigkeiten in eine umfassende Medienkompetenz" und haben dabei den positiven Einfluss von Medienkompetenz auf schriftsprachliche Fähigkeiten im Blick. Medienkompetenz muss heute aber auch dem wirtschaftlichen Aspekt Rechnung tragen, Kinder müssen auch die Marktbedingungen kennen lernen. „Die Einbeziehung ökonomischer Bedingungen [wird, $\mathrm{KH}$ ] in allen Bereichen der Medienkompetenzförderung immer wichtiger werden“ (Marci-Boehncke 2007, 131).

\section{„Emil“ in der Schule}

Klassiker wie Emil und die Detektive bieten sich häufig für den Unterricht an, da sie den Kindern - vor allem aber den Lehrenden - oftmals aus privaten Rezeptionskontexten bekannt sind. Es gibt daher eine Reihe von Unterrichtsvorschlägen zu Emil und die Detektive, z. B. ein Unterrichtsmodell von Kerstin Sterz (2003) oder Vorschläge zur Arbeit an Emil und die Detektive von Ute Frey (2003). Beide schlagen einen Vergleich Buch und Film vor. Frey (ebd., 133) weist daraufhin, dass ein Vergleich zweier gedruckter Texte für Grundschulkinder aufgrund unzureichender Lesefähigkeit ein Problem darstellen kann. Ein Vergleich Buch und Film biete sich deshalb aufgrund des positiven Einflusses von „television literacy“ auf die Lesekompetenz (laut amerikanischen Untersuchungen) an (ebd., 133f.). Außerdem haben Kinder im Bereich ,audiovisueller Medien“ - häufig schon aus der Vorschulzeit (vgl. Marci-Boehncke/Rath 2007) - erstaunliche Kenntnisse. Deshalb kann an Filmen angeknüpft werden, insbesondere wenn schriftsprachliche Kenntnisse noch nicht für einen Vergleich zweier (schriftsprachlicher) Texte ausreichen.

Frey (2003, 143f.) schlägt vor, mit Figurvergleichen zu arbeiten. Zum Beispiel könnte man den Film von 2001 mit dem Roman vergleichen und herausfinden, inwiefern die Figur der Pony Hütchen verändert wurde. Dabei können dann die Unterschiede des geschriebenen Textes zur Filmfassung deutlich werden, z. B. wenn es um den Vergleich von Figurenrede geht. Die Auswahl des Mediums, das den Bedürfnissen des Nutzers entspricht, kann so geübt werden: „Auf diese Weise trägt das vergleichende Vorgehen auch dazu bei, Selektions- und Kombinationsfähigkeiten im Hinblick auf die Mediennutzung der Kinder zu auszubilden." (ebd.) Themen wie Freundschaft, Solidarität und Stärke der Gruppe können über unterschiedliche Medien transportiert werden, um die unterschiedlichen Wahrnehmungskanäle anzusprechen. So besteht die Möglichkeit, dass der Stoff, wenn auch über unterschiedliche Medien, die Kinder mit ihren unterschiedlichen Fähigkeiten erreicht.

\section{Fazit}

Zusammenfassend lässt sich sagen, dass die Vermarktungsstrategie, die bei Emil und die Detektive Anfang der 1930er Jahre verfolgt wurde, als erstaunlich modern und mit heute üblicher Vermarktungsstrategie vergleichbar bezeichnet werden kann. Insbesondere die Verweisfunktion, die jedes Medium auf ein anderes hatte, war eine geschickte Art, Werbung zu machen. Kästners Kenntnisse und Fähigkeiten im Bereich medialer Kommunikation und medialer Vermarktung, kombiniert - was nicht übersehen werden darf mit der Qualität des Stoffes, halfen, dass Emil und die Detektive ein Klassiker wurde, der heute noch beliebt ist wie zu Beginn seiner Vermarktungsgeschichte Anfang des 20. Jahrhunderts. Das Erstaunliche dabei ist, dass dieser Text auch vor bildungsbürgerlichen Bewertungskategorien als Qualitätsstoff gilt - stünden doch andererseits gerade die bei Kästner beschriebenen komplexen Verweisstrukturen zu Werbezwecken unter dem Generalverdacht Adornoscher Kulturkritik. 
Im Hinblick auf den Unterricht sei nochmals mal betont, dass der Umgang mit Medienverbünden seinen Platz in der Schule haben sollte mit dem Ziel, bei den Kindern über unterschiedliche Anknüpfungspunkte Medienkompetenz auszubilden. Emil und die Detektive bietet im Hinblick auf den Unterricht eine Vielzahl von Möglichkeiten der Arbeit am medialen Text, ob nun in Form von Roman oder Film.

\section{Literatur}

Anz, Thomas (Hrsg.) (1998): Erich Kästner. Trojanische Esel. Theater, Hörspiel, Film. München, Wien, Carls Hanser Verlag.

Frey, Ute (2003): Verfilmte Figuren - Hindernisse oder Begleiter auf dem Weg zum Buch? In: Hurrelmann, Bettina/Becker, Susanne (Hrsg.): Kindermedien nutzen. Medienkompetenz als Herausforderung für Erziehung und Unterricht. Weinheim und München, Juventa.

Gast, Wolfgang/Marci-Boehncke, Gudrun (1996): Medienpädagogik in der Schule. Plädoyer für ein fachspezifisches Curriculum jetzt. In: Medien praktisch 20, H. 79, S. 4751.

Haack, Stephanie (2007): Erich Kästners Kinderbuchklassiker Emil und die Detektive und seine ausländischen Ausgaben. Hausarbeit. Johannes-Gutenberg-Universität Mainz.

Harbusch, Ute (Bearb.) (1999): Emil, Lottchen und der kleine Mann. Erich Kästners Kinderwelt. Marbacher Magazin 86/1999 für die Ausstellung im Schiller-Nationalmuseum Marbach am Neckar vom Februar bis April 1999 zum 100. Geburtstag von Erich Kästner am 23. Februar 1999. Marbach/Neckar: Deutsche Schillergesellschaft.

Hasebrink, Uwe/Mikos, Lothar/Prommer, Elisabeth (Hrsg.) (2004): Mediennutzung in konvergierenden Medienumgebungen. Konstanz.

Hoffmann, Hilmar (1995): 100 Jahre Film. Von Lumière bis Spielberg. Düsseldorf: ECON.

Hurrelmann, Bettina/Becker, Susanne (2003) (Hrsg.): Kindermedien nutzen. Medienkompetenz als Herausforderung für Erziehung und Unterricht. Weinheim und München, Juventa.

Lutz-Kopp, Elisabeth (1993): „Nur wer Kind bleibt ...". Erich Kästner-Verfilmungen. Frankfurt, Bundesverband Jugend und Film e.V.

Marci-Boehncke, Gudrun (2006): Intermedialität - Der Rezipient gestaltet das Medium. In: Marci-Boehncke, Gudrun/Rath, Matthias: BildTextZeichen lesen. Intermedialität im didaktischen Diskurs. München: kopäd, S. $13-26$.
Marci-Boehncke, Gudrun (2007): „global kickers“. Die Wilden Fußballkerle als Weltmarke. In: Josting, Petra/Maiwald, Klaus (Hrsg.) (2007): kjl\&m 07.extra. Kinder- und Jugendliteratur im Medienverbund. München, Kopäd. S. 131-142.

Marci-Boehncke, Gudrun/Rath, Matthias (2007): Medienkompetenz für ErzieherInnen. München: kopäd.

Schmid, Johannes (2000): Erich Kästner Verfilmungen und ihre Remakes. Hausarbeit. Ludwig-Maximilians-Universität München.

Sterz, Kerstin (2003): Erich Kästner. Emil und die Detektive. Ein Unterrichtsmodell. Hrsg.: Johannes Diekhans. Paderborn, Schöningh Verlag im Westermann Schulbuch Verlag.

Tornow, Ingo (1998): Erich Kästner und der Film. München, dtv.

Wagner, Ulrike/Theunert, Helga (2006): Neue Wege durch die konvergente Medienwelt. München: Verlag Reinhard Fischer.

\section{URL}

Pressemappe Erich Kästner. http:// www.cecilie-dressler.de/fileadmin/verlags gruppe-oetinger.de/pdf/autoren/3468.pdf 


\section{„Musik gefällt jedem, darum ..." - Medienverhalten und Medienkritik jugendlicher Musikexperten}

\author{
NiNA KÖBERER \& STEFANIE ROSE
}

Die hier dargelegte Analyse über jugendliches Handeln mit und über Musik stützt sich auf die Daten der Ravensburger Jugendmedienstudie (RJMS). ${ }^{1}$ Die Studie hat u. a. Mediennutzungspräferenzen von Jugendlichen erfasst und dabei auch nach dem Medienexpertentum der Jugendlichen gefragt. Im Folgenden wird nur auf den Bereich jener Jugendlichen fokussiert ausgewertet werden, die Musik als ihr Lieblingsmedium angegeben haben und sich selbst als Musikexperten einschätzen. Die Studie greift damit den im Rahmen der Grounded Theory formulierten Anspruch auf, die Objekte sozialwissenschaftlicher Forschung als Experten zu verstehen und aus dieser Expertise von ihnen Aufklärung über ihr Verstehen, Rezipieren und Handeln zu erfragen (vgl. Marci-Boehncke/Rath 2007, 236, dazu Glaser/Strauss 1967; Strauss/ Corbin 1996; Krotz 2005).

Die RJMS kam zu dem Ergebnis, dass Musik unisono über alle Schularten hinweg im gesamten Spektrum medialer Möglichkeiten von Jugendlichen genutzt wird und somit omnipräsent ein integrierter Bestandteil des Alltags Heranwachsender ist (vgl. MarciBoehncke/Rath 2007, 28). Als Begleiter übernimmt Musik neben Motiven der Stimmungsregulation und der Entspannung (vgl. Schramm 2005, 233) im Prozess der Persönlichkeitsentwicklung und Sozialisation auch identitätsstiftende Funktionen (vgl. Wegener 2004b, 22). Dabei steht Musik nicht nur in der Adoleszenz an erster Stelle der Medientätigkeiten (vgl. Barthelmes/Sanders 2001, 95), sondern sie bestimmt auch die Agenda der Gesprächsthemen Jugendlicher. Hier werden Stimmungen und Gefühle diskutabel und in der aktiven Rezeption ausagierbar (vgl. Münch 2002, 72). Musik ist quasi der Metatext, der die Gruppenmitglieder verbindet und über den jedes Gruppenmitglied die Möglichkeit hat, seine eigenen Themen und sich selbst wieder $\mathrm{zu}$ finden und stellvertretend über den Song zu diskutieren.

Setzt man - wie heute üblich - einen erweiterten Textbegriff voraus, dann wird die Nutzbarkeit medienwissenschaftlicher Forschung auch über Musik für erzieherische und pädagogische Kontexte plausibel. Texte als Ausgangsbasis für Gespräche sind zentrales Thema des Deutschunterrichts. Vor allem vor dem Hintergrund der bildungspolitischen Entscheidung, dem Fach Deutsch die Funktion des Leitfachs für Medienerziehung an deutschen Schulen zuzuweisen, ist die Beschäftigung mit der Anschlusskommunikation (Marci-Boehncke/Rath 2007, 228ff.) Jugendlicher über ihr favorisiertes Medium unerlässlich, sucht man einen Unterricht, der an die Lebenswelt der Schülerinnen und Schüler anknüpft. Allerdings steht für die Schülerinnen und Schüler selbst Musik nicht als ein Text in Konkurrenz zu anderen Texten - wie etwa Schrifttexten in Büchern -, sondern Musik ist viel stärker in den Alltag eingebaut (vgl. Vowe/Wolling 2004, 287).

Musik läuft nebenbei, ist Begleitmedium und dabei Teil einer umfassenden Medienkonvergenz, in der die Jugendlichen rezipieren, agieren, kritisieren etc. (vgl. Theunert/Wagner 2006). Diese Medienkonvergenz als ein Konglomerat aus Produktions-, Produktund Rezeptionsperspektiven und -aspekten hat - so der amerikanische Medienwissenschaftler Henry Jenkins (2007) - einen kulturellen Paradigmenwandel herbeigeführt: nämlich denjenigen vom „Zugang“ (access) zur „Beteiligung“ (participation). Dieser Wechsel entspricht der stärkeren Beachtung der Rezeptionskontexte anstelle der rezipierten Ausgangstexte. In dem Moment, in dem der Zugang grundsätzlich kein Problem mehr ist, fokussiert die (auch wissenschaftliche) Betrachtung darauf, wie mit den Angeboten (individuell) umgegangen wird. Die hier vorzustellende Untersuchung jugendlicher Anschlusskommunikation von Musik als Teil der Jugendkultur muss deshalb dieses konvergente Gefüge mit in den Blick nehmen - also neben Fragen nach dem individuellen Mood Management (vgl. Schramm 2005) auch die Mediennutzung im Medienverbund und die Medienkritik berücksichtigen.

War für Dieter Baacke (1996) Medienkritik ein Teilbereich der Medienkompetenz (neben Mediengestaltung, Medienkunde und Mediennutzung), so weist Stefan Aufenanger (2006) darauf hin, dass Medienkritik als Querschnittsbereich alle Aspekte der Medienkompetenz durchziehen müsse. Seine sechs Teilkategorien decken dabei die Themenfelder auch schulischer Medienkompetenzförderung ab.

Um nun einen möglichst vom Schüler ausgehenden Unterricht zu planen, muss man sich mit den Nutzungsmustern und der Reflexionsfähigkeit von Jugendlichen über ihre Medien auseinander setzen.

- Wir schauen uns zunächst an, was Jugendlichen ihre Musik bedeutet und wie sie sie reflektieren. Die Frage, was Jugendlichen Musik bedeutet, haben auch Barthelmes/Sander (2001) in ihrer Studie Erst die Freunde dann die Medien gestellt und haben aus den Antworten zehn Nut- 


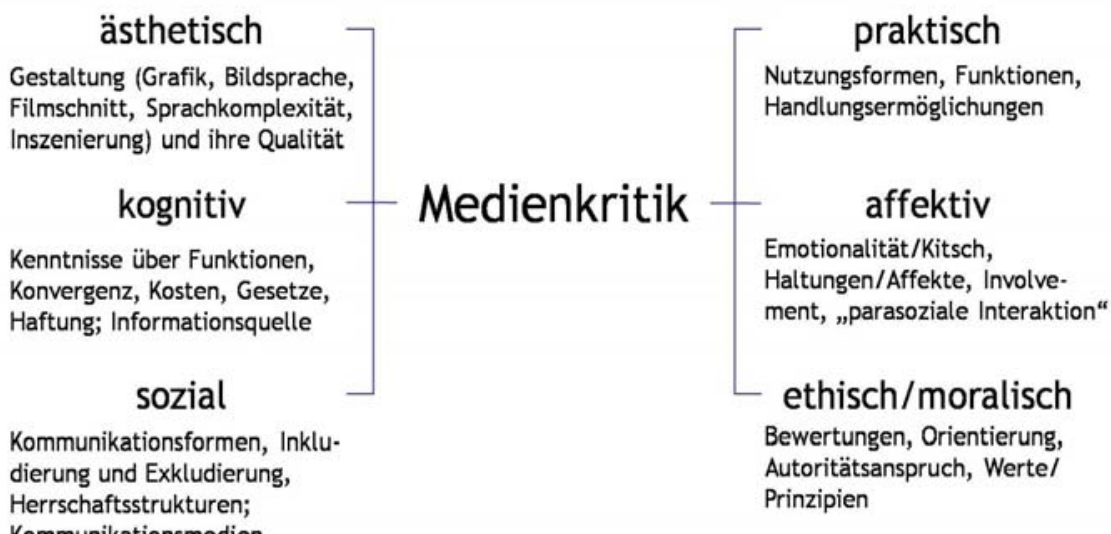

Kommunikationsmedien

(Geld, Macht, Anerkennung ...)

Medienkritik als Grundkategorie (Aufenanger 2006)

zungsmuster erarbeitet. Wir werden neun dieser zehn Nutzungsmuster mit den Daten der stärker quantifizierenden RJMS näher in den Blick nehmen. Vieles finden wir wieder, etwas können wir noch ergänzen und manches scheint in Veränderung begriffen.

- Dann werden wir ein weiteres Nutzungsmuster ausführlich darstellen. Medien scheinen uns ein wichtiges Feld, auf dem Jugendliche ihre moralischen Kategorien schärfen. Anders als häufig diskutiert, sind die Jugendlichen aber nicht in einer simplen Ursache-Wirkungs-Verschränkung den normativen Angeboten der Medien ausgeliefert. Dies wird gerade an den Medienstars der Jugendlichen deutlich. Jugendliche sehen ihre medialen Stars durchaus auch kritisch. Sie argumentieren bzw. reflektieren dabei wertegestützt und lassen ihre Haltungen und Einstellungen erkennen. Anschließend betrachten wir dieses Verhältnis der Jugendlichen zu ihren Musikstars und untersuchen, wie das jugendliche Reflexionsvermögen aussieht. Was erfahren wir über die Starkritik zu jugendlichen Wertgefügen?

- Schließlich fokussieren wir noch stärker auf die Anschlusskommunikation über Musik. Wir wollten wissen, welches Reflexionsvermögen Jugendliche zu Gewalt in Musik zeigen und inwiefern sie Musik - gerade im Medienverbund - auch als „Ware“ begreifen. Welches Bewusstsein lassen sie über ökonomische Strukturen des Musikmarktes erkennen, welche sind für sie handlungsrelevant, welche können sie auch kritisch reflektieren? Medienkonvergenz ermöglicht dabei quasi strukturell eine Meta-Ebene, da sich sowohl über die Tonträger unterhalten wird als auch über die Inhalte von Songs. Markt, Macht und Medien sind auch in jugendlichen
Lebenswelten von Interesse und prägen Gespräche und Kommunikation.

\section{Nutzungsmuster von Musik: Was bedeutet Musik für die Jugendli- chen}

Bei Barthelmes/Sander (2001) finden sich insgesamt zehn Kategorien (siehe Kasten), welche jugendlichen Umgang mit Musik genauer in den Blick nehmen. Dass Jugendliche Musik zunächst ,alleine für sich“ rezipieren, ist, wie zu erwarten, auch durch die Daten der RJMS zu belegen. Der heimische Musikkonsum ist mit 79,6\% höchst präferiert. Dabei läuft Musik nebenbei (Berger 2001) und ist integriert in den ganzen Tagesablauf. Wenn Jugendliche Musik hören, singen sie häufig ihre Songs mit (56,84\%). Darin unterscheiden sich Jungen und Mädchen nicht. Mädchen tanzen jedoch signifikant öfter zu Musik als Jungen.

1. Die Jugendlichen hören Musik vor allem ,alleine für sich“.

2. Musik spendet Trost und verstärkt Stimmungen und Gefühle.

3. Für sich Musikhören ist ein Dialog mit dem Lieblings-Star.

4. Die Jugendlichen möchten die Musiktexte verstehen.

5. Die Jugendlichen möchten „,in“ sein, die Jugendlichen möchten einem Stil treu bleiben - manchmal sind harte Schnitte unvermeidlich.

6. Die Jugendlichen grenzen sich mit Musikstilen vom Geschmack anderer Jugendlicher sowie vom Geschmack ihrer Eltern oder Geschwister ab.

7. Musik ist ein soziales Medium in der Gleichaltrigengruppe.

8. Die Musikvorlieben dienen der Teilhabe an jugendkulturellen Szenen.

9. Musikstücke und Liedtexte werden zum Lebens-Motto.

10. Musik ist ein „Anker im Meer der Biographie“.

Nach Barthelmes/Sander (2001, 100-109). 
Offene Antworten wie „Gefübl. Muss alles stimmen" (Pb. 160) oder „Sie gibt mir Kraft und hilft mir Probleme zu lösen. Manchmal bin ich total unglücklich - dann böre ich Musik und es geht mir

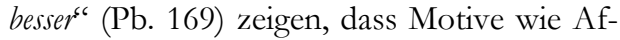
fektkontrolle und Ausdruck von Autonomie und Lebensstil erwartungsgemäß eine hohe Bedeutung haben (vgl. Münch 2002, 74). Wie Barthelmes/Sander in ihrer zweiten Kategorie betonen, leistet Musik mood managment und dient damit als Unterstützung in der jugendlichen Entwicklungsphase. Insgesamt gibt der Großteil der Jugendlichen (rund $90 \%$ ) an, dass Musik ihre Stimmung beeinflusst. Musik ist das Medium, welches überall von den Jugendlichen als Mittel zur Stimmungsoptimierung beziehungsweise Stimmungsverstärkung genutzt wird. Dabei intensiviert sie positive und negative Gefühle oder dient zur kathartischen Verarbeitung (vgl. Zillmann 1988). Durch Musik erzeugte Gefühle der Zuneigung, Liebe und Sehnsucht richten sich auch in Form von Projektionen auf einzelne Stars.

Musik wird zum Dialog mit dem Lieblingsstar, wie es Barthelmes/Sander durch ihre dritte Kategorie formulieren. Beim Musikhören im eigenen Zimmer entwickeln Jugendliche im Dialog mit ihren Stars Probegefühle und Muster von Probehandeln, ohne Angst vor Sanktionierung. Die alleinige Musikrezeption hilft bei der Suche nach sich selbst, kultiviert das Verhältnis zum eigenen Star und hilft unterschiedliche Situationen zu imaginieren. Um sich dem Star ,noch näher“" zu fühlen, findet oft ein Rückgriff auf Fanartikel wie TShirts, Bettwäsche oder Poster statt. Die Sehnsucht nach dem Star ist somit auch mit einem Konsumbedürfnis verbunden.

Neben der Melodie der Songs, welche die Gefühle der Jugendlichen ansprechen, sind auch die Songtexte von erheblicher Relevanz, wie Barthelmes/Sander in Punkt 4 festhalten. Rund $75 \%$ der befragten Jugendlichen gaben an, dass ihnen auch die Songtexte bei der Musikrezeption von Bedeutung sind. Sie korrespondieren häufig mit der Gefühlssituation oder sind komplementär. Auf jeden Fall sind sie den Jugendlichen nicht egal. Lieder werden ausgewählt oder abgelehnt mit Kenntnis der Liedtexte. Dabei geht die Präferenz der Sprache (Muttersprache bzw. Fremdsprache) eindeutig zugunsten der Fremdsprache mit rund $90 \%$. Gerade weil die Songtexte verstanden werden wollen, stehen die Jugendlichen vor der Notwendigkeit der Übersetzung, wobei diese unter $\mathrm{Zu}$ hilfenahme des Medienverbundes erfolgt. An dieser Stelle zeigt sich genderspezifisches Medienverhalten deutlich. Jungen nutzen zur Übersetzung häufiger das Internet (25,4 \%), während Mädchen dazu auf Zeitschriften zurückgreifen (17,19 \%) (vgl. MarciBoehncke/Rath 2007, 84).

Insgesamt lässt sich festhalten, dass Jugendliche auch einen kognitiven Umgang mit Musik pflegen und sie nicht nur als reines Gefühlsmedium wahrnehmen. Die harten Schnitte, wie sie Barthelmes/Sander in ihrer 5. Kategorie verzeichnen, sind bei den in der RJMS befragten Jugendlichen eher unterrepräsentiert. Bei den Probanden ist der Mainstream beliebt - Pop und Hip Hop gelten als die beliebtesten Musikrichtungen. „Extremere“ Musikpräferenzen wie Punk, Rechtsrock oder Heavy Metal sind dabei eher unterrepräsentiert.

Die von Barthelmes/Sander aufgestellte These, dass sich Jugendliche bewusst durch das Medium Musik von ihren Eltern abgrenzen, kann in dieser Absolutheit nicht durch die Daten der RJMS belegt werden. Obzwar die Jugendlichen Musik nicht mit ihren Eltern rezipieren, scheint die weiterführende Frage vielmehr, ob nur die Rezeption allein erfolgt oder ob damit auch eine grundsätzliche Kontraposition zum Musikstil der Eltern beziehungsweise Geschwister gegeben ist. Da Geschwister sowie auch Eltern als Gesprächspartner über Musik angegeben werden und sie auch diejenigen sind, die Musikwünsche (CDs) erfüllen, scheint eine inhaltliche Abgrenzung bei vielen Jugendlichen nicht mehr unbedingt identitätsstiftend $\mathrm{zu}$ sein. Die Rezeption erfolgt jedoch allein, hier wollen die Jugendlichen nicht beobachtet werden - auch wenn sie vielleicht die gleichen Songs hören wie ihre Eltern. In der Zukunftsgesellschaft, wie die amerikanische Ethnologin Margarete Mead (1971) das Verhältnis der modernen Generationen charakterisierte, bleiben auch die Elterngenerationen - stärker als etwa in den 1950er/1960er Jahren - an der aktuellen jugendorientierten Musik interessiert. Das macht eine inhaltliche Abgrenzung der Jugendlichen immer schwerer, da ihre Eltern ebenfalls Fans der Songs der Moderne sind. Die Distinktion erfolgt daher vor allem lokal, nicht thematisch. Ein Bedauern, dass dadurch ein Mittel zur Abgrenzung verloren ginge, ist somit nicht zu verzeichnen. Die Rezeptionssituation ist privat, kommuniziert wird mit Freunden und dennoch dürfen die Interessen die Gleichen sein (über $70 \%$ fänden es sogar gut, wenn die Eltern die gleichen Interessen wie sie selbst hätten). An dieser Stelle stehen die Ergebnisse der Studie konträr zu den Daten von Barthelmes/Sander.

Ganz anders die Ergebnisse, welche sich mit der These von Barthelmes/Sander Musik ist ein soziales Medium in der Gleichaltrigengruppe in Verbindung bringen lassen. In der PeerGroup ist Musik wichtigstes Thema auf der 
Gesprächsagenda. Es lässt die Jugendlichen sich selbst thematisieren, ohne dass sie sich als Person explizit der Diskussion und Selbstreflexion aussetzen müssen.

Die Musik steht pars pro toto. Neben den Freunden werden Geschwister und Eltern/ Verwandte noch als Kommunikationspartner gesucht, Lehrer sind nicht gefragt. Dies scheint u. E. im Wesentlichen eine Vertrauensfrage zu sein. Musik ist für die Jugendlichen auch identitätsstiftend. Neben alleiniger Musikrezeption steht die Rezeption mit $37 \%$ mit Freunden an zweiter Stelle. Über Musik drücken die Jugendlichen ihre Persönlichkeit aus. Es ist deshalb nicht verwunderlich, dass es ihnen wichtig ist, dass auch die Freunde den gleichen Musikgeschmack haben und dies ist auch real gegeben (51\% gleicher $\mathrm{Mu}$ sikgeschmack, $47 \%$ teilweise). Das garantiert emotionale Sicherheit und Ähnlichkeit.

Bei vielen Jugendlichen stimmen die Musikvorlieben mit der Teilnahme an jugendkulturellen Szenen überein (s. Kategorie 8 bei Barthelmes/Sander). So sind das Lebensgefühl und der Stil der Stars von Wichtigkeit. Musik stellt in diesem Zusammenhang, neben Kleidung und Sprache auch ein Medium dar, um jugendkulturelle Szenen zu entwerfen. Musikstücke und Liedtexte können auf diese Weise vorübergehend zum wichtigen Lebensmotto werden, wie es Barthelmes/ Sander in ihrer neunten Kategorie formulieren. Musik spricht Jugendliche sowohl auf der emotionalen als auch auf der kognitiven Ebene an. Während 37,43 \% der Jugendlichen großen Wert auf die Melodie und 21,39 $\%$ auf den Rhythmus eines Songs legen und sich somit von den Musikstücken auf der Gefühlebene angesprochen fühlen, vertreten $27,27 \%$ der Probanden die Meinung, dass der Songtext und somit die inhaltliche Aussage eines Songs am Wichtigsten sei. Die 10. These bei Barthelmes/Sander (10. Musik ist ein Anker im Meer der Biographie), also die biographische Verankerung eines Musikstücks, an dem sich Lebenserinnerungen festmachen, haben wir nicht eigens thematisiert und können wir daher auch nicht diskutieren.

\section{Starkritik: Wie stehen Jugendliche zu ihren Stars?}

Wir gehen davon aus, dass neben dem Mood Management, also dem Umgang mit den eigenen Gefühlen, das Probehandeln und das moralische Ausdifferenzieren über die Auseinandersetzung mit Musik einen weiteren wichtigen Nutzungsgrund darstellen. Aus diesem Grund haben wir in Anlehnung an die von Barthelmes/Sander vorgeschlagenen Nutzungsmuster von Musik eine weitere Kategorie gebildet. Wir stellen folgende 11 . These auf:
Musik bietet die Möglichkeit zur Ausbildung von Wertebewusstsein und der Fähigkeit, Werthaltungen reflektieren zu können.

Da mediale Bezugspersonen als Bausteine eines Identitäts-Scripts (vgl. Paus-Haase 1999, 170) dienen, suchen Jugendliche sich Vorbilder und orientieren sich stark an ihnen. Diese medialen Bezugspersonen werden jedoch nicht nur bewundert und imitiert, sondern durch Jugendliche auch kritisiert, bewertet und beurteilet (vgl. Wegener 2004a, 23). Die Tatsache, dass Jugendliche über ihre Stars nachdenken, über sie sprechen und sich kritisch mit ihnen auseinandersetzen, zeugt davon, dass Musik dazu dienen kann, ein kritisches Reflexionsvermögen und eine werturteilende Argumentationskompetenz zu entwickeln. Die Ausbildung von Werturteilskompetenz setzt voraus, dass Sachverhalte analysiert und bewertet werden und Urteile argumentativ vertreten werden können. Urteilsfähigkeit wird hierbei verstanden als „kognitive Kompetenz, die auf der Überzeugung basiert, dass Argumentation interessengeleitet ist" und „,der Einordnung von Aussagen in ein Wertsystem dient" (MarciBoehncke/Rath 2007, 96).

Die Ausbildung der werturteilenden Argumentationskompetenz im Sinne einer kognitiven Kompetenz ist immer an die Analyse und Begründung von Argumentationsangeboten - in diesem Fall an die Kritik der Jugendlichen an ihre Lieblingsstars - gebunden. Diese Kompetenz ist „theoretisch beschreibbar mit Lawrence Kohlbergs Theorie der moralischen Entwicklung (...) und empirisch erfassbar mit einer an ihn angelehnten Analyse jugendlicher Argumentationsformen" (ebd., 42).

Lawrence Kohlbergs kognitive Entwicklungstheorie der Moral konzentriert sich auf die Entwicklung universeller Stufen moralischen Denkens. Bezogen auf ein Modell der Entwicklung beschreibt der Begriff Stufe qualitativ unterschiedliche Etappen in der Entwicklung des Denkens (vgl. Kohlberg 1974, 10). Moralisches Denken baut nach Kohlberg zunächst auf der Berücksichtigung der nutzenbringenden Folgen des eigenen Handelns auf (präkonventionelles Niveau, Stufen 1 und 2) und geht dann über zur Identifikation mit zwischenmenschlichen und gesellschaftlichen Erwartungen (konventionelles Niveau, Stufen 3 und 4). Schließlich wird eine Ebene erreicht, auf der die Menschen ihre eigenen moralischen Prinzipien ausgearbeitet haben (postkonventionelles Niveau, Stufen 5 und 6). Dieser Verlauf der Stufen wird als universell und in seiner Abfolge unveränderlich angesehen. Kohlberg geht es bei seinem Stufenmodell vorrangig um die Struktur des moralischen Urteilens 
und nicht um die kulturell oder individuell verschiedenen Inhalte moralischer Entscheidungen. In diesem Sinne kann ein moralisches Urteil ,als ,moralisch' definiert werden, ohne seinen Inhalt (die beurteilte Handlung) betrachten und ohne überlegen zu müssen, ob es mit den eigenen Urteilen oder Maßstäben übereinstimmt" (Kohlberg 1968, 29). Das zentrale Forschungsinteresse Kohlbergs war demnach die Bestimmung der kognitiven Strukturaspekte des moralischen Denkens und, damit verbunden, die Differenzierung von (invarianten) Struktur- und (variablen) Inhaltsaspekten moralischer Urteile.

Die RJMS hat untersucht, auf welchem Niveau die Jugendlichen moralisch ansprechbar sind, d. h. auf welcher Stufe sie argumentieren. Zudem wurde die inhaltliche Ebene in den Blick genommen. Die materialen Wertvorstellungen der Jugendlichen in Bezug auf ihre Stars wurden analysiert und in Anlehnung an die Kategorien von Kohlberg systematisiert. Wenn sich Jugendliche über Musik unterhalten, ergeben sich - neben CD's und $V i d e o s$ - zwei weitere große Themenfelder: Songs und Bandmitglieder/Stars (vgl. MarciBoehncke/Rath 2007, 83). Die Mehrheit der Jugendlichen gibt an, dass ihnen der Lebensstil, die Denkweise, die Kleidung etc. ihrer Stars wichtig sind.

Die offenen Antworten der Starkritik wurden inhaltsanalytisch in acht Kategorien zusammengefasst: Aussehen, Content, Konsum, Leistung, Nation, Öffentlichkeit, Parasozial und Moral (vgl. ebd., 81). In diesem Zusammenhang ist festzuhalten, dass die Jugendlichen an ihren Stars das Aussehen, das Auftreten in der Öffentlichkeit sowie die Leistung am meisten kritisieren (ebd., 61f.): „Dass er manchmal so eine Afro-Frisur hat, das sieht nicht schön aus" (Pb. 10) oder ,sie kauft sich zu viele Pelze und dann redet sie so ein Zeng wie: die armen Tiere" (Pb. 18).

Die Musikexperten stellen vorrangig äußere Merkmale der Vorbilder in den Fokus der Kritik. Sie sind aber auch in der Lage, das Auftreten und Verhalten der Stars zu reflektieren und mit ihren eigenen Wert- und Normvorstellungen abzugleichen. Die acht Kategorien zur Starkritik sind in einem weiteren Schritt zusammengebündelt und den Stufen der moralischen Argumentationsfähigkeit von Kohlberg zugeordnet worden. Es sollte erfasst werden, auf welcher moralischen Stufe die Jugendlichen argumentieren. Die Kategorien Aussehen, Leistung und Nation sind den geschmacklichen Urteilen zugeordnet worden, welche mit einem Argumentationsvermögen auf Stufe 1 übereinstimmen. Diejenigen, welche die virtuelle Sozialität (Parasozialität, Öffentlichkeit) kritisieren, lassen eine Argumentationsfähigkeit auf den Stufen 2 und 3 erkennen. Bei den Argumenten zu Content, Konsum und Werten, die als Moral zusammengefasst wurden, ist eine Tendenz zur konventionellen und postkonventionellen Orientierung erkennbar.

Bei den Musikexperten ist mit knapp zwei Dritteln der Antworten ,eindeutig Geschmack das beherrschende Moment der Beurteilung“, die virtuelle Sozialität fällt kaum ins Gewicht und der Aspekt der Moral liegt immerhin bei einem Drittel der Antworten (Marci-Boehncke/Rath 2007, 82). Dieses Ergebnis macht deutlich, dass die Beurteilung der Musikstars stark an äußerlichen Kriterien orientiert ist und dementsprechend argumentiert der Großteil der jugendlichen Musikexperten auf Stufe 1.

\section{Anschlusskommunikation: Wie orientieren sich Jugendliche im konvergenten Medienumfeld?}

Kinder und Jugendliche sind auch ökonomisch ein wichtiger Faktor im Medienverbund. Laut der aktuellen Kids Verbraucheranalyse 2008 des Ehapa-Verlags stehen in Deutschland allein den 6- bis 13-Jährigen rund 2,3 Mrd. Euro an regelmäßigen Geldzuflüssen oder Geldgeschenken zur Verfügung und rund 3,6 Mrd. Euro liegen als Sparguthaben für die Kids auf der hohen Kante. Zwar stehen Süßigkeiten, Zeitschriften, Comics, Magazine und Eis auf den ersten drei Plätzen der Taschengeldverwendung, aber gespart wird in erster Linie auf „Mediales“, u. a. auf Handy 14 \%, PC, PC-Zubehör und Computerspiel zu je $11 \%$ und auf Konsolenspiele zu $9 \%$. Fan-Sein bedeutet dabei - schon bei den Kids - in erster Linie, dass konvergente Mediengefüge in Bezug auf die inhaltliche Orientierung an Musik zu nutzen und dadurch an der Kommunikation teilhaben zu können. Die Fans bilden eine virtuelle Community, traditionelle Gruppenmerkmale wie Fanartikel als Erkennungszeichen oder eine Clubstruktur als Organisationsebene sind nicht mehr nötig.

In der RJMS geben die Musikexperten folgende Bezugsquellen für den Erwerb von Fanartikeln ihrer Musikstars an: 69,6\% kaufen Zeitschriften, 51,2\% rezipieren Musiksendungen und $48 \%$ suchen im Internet Informationen zu ihren Stars. Die Kommunikation über Musik nimmt einen hohen Stellenwert bei den Jugendlichen ein. Neben den Gesprächen über die Stars und die Starkritik dienen Liedtexte als Grundlage für die Auseinandersetzung mit Musik. Die Musikexperten geben mehrheitlich an, dass sie in den Songtexten Gewalt erkennen. Ein Drittel der Gymnasiasten ist sich darüber bewusst, dass in ihren Lieblingsliedern Gewalt vor- 
kommt. Bei den Realschülern sind es $10 \%$ und bei den Hauptschülern $20 \%$ (vgl. ebd., 85).

Auf die Frage, warum Gewalt in Songs vorkommt, antworten die Jugendlichen zu gleichen Teilen (19 \%), dass die Sänger eigene Erfahrungen abbilden und dass sie warnen wollen. Von den Befragten sind $16 \%$ der Meinung, dass Zeitgeist abgebildet wird. Macht (9\%) und Coolness (6\%) sind weitere Argumente. Immerhin $5 \%$ sind der Meinung, Gewalt werde aus ökonomischen Gründen - als Marketingmittel - zum Thema gemacht. Dies lässt darauf schließen, dass Jugendliche über ein ökonomisches Bewusstsein verfügen und Musik im wirtschaftlichen Kontext verorten, reflektieren und bewerten können.

\section{Fazit}

Von den Musikexperten der RJMS gaben $65 \%$ an, dass sie im Unterricht gerne mehr über Musik erfahren wollen und einer unterrichtlichen Behandlung positiv gegenüber stehen. Nur $11 \%$ der Befragten möchten Musik nicht als Unterrichtsgegenstand, wohingegen $24 \%$ unschlüssig sind, ob sie $\mathrm{Mu}$ sik im Unterricht thematisieren möchten. Gerade die $24 \%$ lassen darauf schließen, dass es ausschlaggebend ist, wie die Thematisierung im Unterricht vonstatten gehen soll. Gespräche über Emotionen sowie Stimmungen, die Reflexion über Stars, die diskursive Auseinandersetzung über Musiktexte und über Themen, die durch Musik vermittelt werden, bieten sich als Ausgangslage für einen Unterricht an, der an die Lebenswelt von Jugendlichen anknüpft. Probleme und Aufgaben der Jugendlichen in der Phase der Allokation können aufgegriffen werden und der Aufbau und die Vermittlung von Einstellungen und Haltungen können durch die diskursive Auseinandersetzung beziehungsweise die argumentative Reflexion und Urteilsfindung am Unterrichtsgegenstand $\mathrm{Mu}$ sik vollzogen werden.

\section{Anmerkung}

1 Alle Daten im folgenden Text ohne anderweitige Quellenangabe sind aus der Ravensburger Jugendmedienstudie RJMS.

\section{Literatur}

Aufenanger, Stefan (2006): Medienkritik. Alte und neue Medien unter der Lupe. In: Computer + Unterricht, H. 64, S. 6-9.

Baacke, Dieter (1996): Medienkompetenz Begrifflichkeit und sozialer Wandel. In: Rein, Antje von (Hg.): Medienkompetenz als Schlüsselbegriff. Bad Heilbrunn: Klinkhardt, S. 4-10.
Barthelmes, Jürgen/Sander, Ekkehardt (2001): Erst die Freunde, dann die Medien. Medien als Begleiter in Pubertät und Adoleszenz. Medienerfahrungen von Jugendlichen. München: Deutsches Jugendinstitut.

Bechdolf, Ute (1999): Puzzling Gender. Reund De-Konstruktionen von Geschlechterverhältnissen im und beim Musikfernsehen. Weinheim: Deutscher Studienverlag.

Glaser, Barney/Strauss, Anselm (1967): The discovery of grounded theory. Chicago: Aldine.

Hoffmann, Dagmar (2002): Attraktion und Faszination Medien: jugendliche Sozialisation im Kontext von Modernisierung und Individualisierung. Münster/Hamburg : Lit-Verlag. Jenkins, Henry (2006): Convergence Culture. Where old and new media collide. New York/London: New York University Press.

Kohlberg, Lawrence (1968): Moralische Entwicklung. In: Althof, Wolfgang (Hrsg.) (1995): Lawrence Kohlberg. Die Psychologie der Moralentwicklung. Frankfurt a. M.: Suhrkamp Verlag, S. 7-40.

Kohlberg, Lawrence: (1974): Zur kognitiven Entwicklung des Kindes. Frankfurt a. M: Suhrkamp Verlag.

Krotz, Friedrich (2005): Neue Theorien entwickeln. Eine Einführung in die Grounded Theory, die Heuristische Sozialforschung und die Ethnographie anhand von Beispielen aus der Kommunikationsforschung. Köln: Herbert von Halem Verlag.

Luca, Renate (2003): Medien. Sozialisation. Geschlecht. : Fallstudien aus der sozialwissenschaftlichen Forschungspraxis. München: kopaed.

Marci-Boehncke, Gudrun/Rath, Matthias (2007): Jugend - Werte - Medien. Die Studie. Unter Mitarbeit von Timo Röcker. Weinheim und Basel: Beltz Verlag

Mead, Margarete (1971): Der Konflikt der Generationen. Jugend ohne Vorbild. Freiburg im Breisgau: Walter

Müller, Renate/Glogner, Patrick/Rhein, Patrick (2002): Wozu Jugendliche Musik und Medien gebrauchen. Jugendliche Identität und musikalische und mediale Geschmacksbildung. Weinheim/München: Juventa Verlag.

Münch, Thomas (2002): Musik, Medien und Entwicklung im Jugendalter. In: Müller, Renate/Glogner, Patrick/Rhein, Patrick (Hrsg.): Wozu Jugendliche Musik und Medien gebrauchen. Jugendliche Identität und musikalische und mediale Geschmacksbildung. Weinheim/München: Juventa Verlag. 
Paus-Haase, Ingrid (1999): Medienhandeln und Kinder-Peergroups im lebensweltlichen Zusammenhang. In: Fromme, Johannes/ Krommer, Sven/Mansel, Jürgen/Treumann, Klaus-Peter (Hrsg.): Sozialisation. Kinderkultur und Mediennutzung. Opladen: Leske und Budrich. S. 161-182.

Schramm, Holger (2005): Mood-Managment durch Musik. Köln: Herbert von Halem Verlag.

Strauss, Anselm/Corbin, Juliet (1996): Grounded Theory: Grundlagen qualitativer Sozialforschung. Weinheim: Psychologie Verlags Union/Beltz.

Theunert, Helga/Wagner, Ulrike (2006): Konvergenz als neue Komponente im Medienalltag Jugendlicher. Empirische Befunde und Relevanz für die Orientierungsfunktion von Medien. In: Marci-Boehncke, Gudrun/ Rath, Matthias (Hrsg.): Jugend - Werte Medien: Der Diskurs. Weinheim und Basel: Beltz Verlag. S. 57-69.

Vowe, Gerhard/Wolling, Jens (2004): Radioqualität - was die Hörer wollen und was die Sender bieten: Vergleichende Untersuchung zu Qualitätsmerkmalen und Qualitätsbewertungen von Radioprogrammen in Thüringen, Sachsen-Anhalt und Hessen. München: kopäd.

Wegener, Claudia (2004a): Identitätskonstruktion durch Vorbilder. Über Prozesse der Selektion, Aneignung und Interpretation medialer Bezugspersonen. In: Merz 48, H. 6, S. 20-31.

Wegener, Claudia (2004b): Manipulation oder Zeitgeist? Lesarten populärer Musik. In: Fritz, Karsten/Sting, Stephan/Vollbrecht, Ralf (Hrsg.): Mediensozialisation. Pädagogische Perspektiven des Aufwachsens in Medienwelten. Opladen: Leske und Budrich. S. 61-74.

Zilmann, Dolf (1988): Mood Managment. Using Entertainment to Full Advantage. In: Donohew, Lewis (Hrsg.): Communication. Social Cognition, an Affect. Hillsdale: Erlbaum. 


\section{„Wag the Dog“ und Medien- wirkung}

\section{PETER SCHMITT}

\section{Einleitung}

„Wenn alles verloren zu sein scheint ... zieh in den Krieg. Das ist die klassische Antwort auf unlösbare Probleme im Inland. Suchen Sie sich jemanden, der den Instinkt, den Stil und die künstlerische Fähigkeit besitzt, einen Krieg zu erschaffen, den Amerika lieben kann - im Fernsehen. Dann werden Sie siegen.“ (Beinhart 2003, 165)

Conrad Brean, gespielt von Robert de Niro, ist in dem Film Wag the Dog der Mann, der diesen Krieg erschaffen kann. Als graue Eminenz im Wahlkampfteam des Präsidenten ist er für den Erfolg des medialen Wahlkampfes verantwortlich. Ihm gelingt es die filmische Öffentlichkeit von der Katastrophe des präsidialen Kinderschänderskandals mit einem medial inszenierten Krieg abzulenken und rettet letztendlich so dem amerikanischen Präsidenten die Wiederwahl. Ist das nun ein aberwitziger Einfall der Drehbuchautoren David Mamet und Hilary Henkin oder ein ernst zu nehmendes Szenario, dass „nicht soweit hergeholt ist wie es scheint ${ }^{\text {"2 } 2 ?}$

Die gewollte medienzentrierte Betrachtung der filmischen Koexistenz von Medien und Öffentlichkeit beantwortet keine Fragen. Sie gibt jedoch sicherlich ein sozialkritisches Statement der Filmemacher wieder. Die Bevölkerung wird einfach nicht gezeigt. Das Verhältnis zwischen Politik und Medien hingegen trägt die Story. Was in den Medien erscheint, wird von der filmischen Öffentlichkeit als real wahrgenommen. Ist es nun tatsächlich so, dass wir Rezipienten alles unreflektiert schlucken, was uns die Medien so auftischen?

Während „,die Grundthese der simplifizierenden Medienkritik lautet: Die Medien können alles, wir nichts“ (Rath 2006, 122; vgl. auch zur sog. These von der ,starken Wirkung“ der Medien Rath 2000), ist dennoch das satirische Moment in diesem übertrieben auskontrastierten Verhältnis zwischen manipulierbarer Öffentlichkeit und „Allmacht der Medien“ spätestens seit der realen, filmähnlichen „Lewinsky-Affäre“ (1998) und der in der breiten Öffentlichkeit als inszeniert geltenden „Jessica Lynch-Story“ (2004) zumindest teilweise relativiert worden. Politische Ereignisse werden in der Tat oft in den Medien erst zum eigentlichen Ereignis gemacht und andere, vermeintlich wichtigere, werden vernachlässigt. Von manchen Ereignissen wird wochenlang berichtet, andere wiederum werden von den Medien völlig ignoriert. Es gibt Nachrichten, die an erster Stelle stehen, die sozusagen eine „Lead story“ darstellen und dann wieder welche, von denen nur am Rande zu hören ist. Manche Ereignisse werden medial auf eine bestimmte Art und Weise verpackt, andere erscheinen ungeschönt in dokumentarischem Stil.

Es sind die Medien und deren Berichterstattungen, die oft den großen Unterschied ausmachen zwischen dem, was die breite Bevölkerung in ihre eigene Themenstruktur einflicht und was nicht. Somit sind die Medien ein mächtiger Bestandteil als Verbreiter, als "öffentlich Macher" von Politik. Hier ist die Themensetzung (Agenda Setting) ein besonderer Faktor in dem bereits Jahrhunderte alten Kanon aus Politik, Medien und Öffentlichkeit. In der vorliegenden Arbeit werde ich zunächst das medienwissenschaftliche Phänomen des „Agenda Setting“ beschreiben um daraufhin diese Beschreibung mit Fallbeispielen aus dem Film Wag the Dog anzureichern. Beenden möchte ich die Arbeit mit einem Fazit, dass sich unter anderem kurz mit der „Ökonomie der Aufmerksamkeit“ von Georg Franck beschäftigen wird.

An diversen Stellen sind Bezüge zum Film mit dem von mir erstellten Sequenzplan und mit Filmausschnitten aus dem Videoportal Youtube verlinkt.

\section{Agenda Setting / Wag the Dog}

„Vom Golfkrieg haben wir kollektiv die selben Bilder. Sonst nichts. Eine Kontrolle war unmöglich. Die Bilder hätte man auch fälschen können. So kamen David Mamet und ich darauf, darüber zu diskutieren, wie viel man fälschen könnte. Kommt es später raus, ist das egal. Da gibt es schon was Neues!“33

Auf den ersten Blick spricht Barry Levinson über die fehlende Möglichkeit des Rezipienten im Fernsehen gesehene Bilder auf ihren Wahrheitsgehalt prüfen zu können. Auf den zweiten Blick jedoch ist hier von einem ganz anderen Phänomen die Rede: Das medienwissenschaftliche Phänomen des „Agenda Setting“. Denn die Tatsache, dass „später“ die Wahrheit über die Fälschung „rauskommt", bedeutet noch lange nicht, dass sie von den Rezipienten wahrgenommen wird bzw. als wichtig wahr genommen wird, da tagtäglich „was Neues“ auf der Tagesordnung/Agenda der Medien steht und eine skandalöse politische Inszenierung von gestern deswegen leicht in „der Vielfalt des Informationsgewitters" (Rath 2006, 122) untergehen kann.

\section{Platzierung und „Lead stories"}

Innerhalb dieser Agenda findet eine Platzierung von Themen nach einer Rangfolge statt. Was auf der Titelseite steht oder was als Ers- 
tes in den Fernsehnachrichten gesendet wird, beeinflusst den „Grad an Wichtigkeit, der einem Thema im öffentlichen Diskurs beigemessen wird.“ (Rössler 1997, 17) „Diejenigen Themen der politischen Diskussion, welche die Medien hervorheben, werden in der Folge auch von den Rezipienten als wichtig betrachtet.“ (Maletzke, zit. nach ebd.) Bernard Cohen erläutert diesen Umstand folgendermaßen: „The press [..] may not be successful much of the time in telling people what to think, but it is stunningly successful in telling its readers what to think about." (Cohen, zit. nach ebd., 23) Die Agenda-Setting-Theorie, auch „Tagesordnungs-“ oder „Thematisierungsfunktion" der Medien genannt, beruht also auf dem Gedanken, dass Massenmedien nicht so sehr beeinflussen, was, sondern worüber wir nachdenken sollen (vgl. McCombs in ebd., 16).

Der Ansatz des „Agenda Setting“ ist nun zunächst eine Hypothese über nichtpersuasive Medienwirkungen (vgl. ebd., 10). Der Rezipient „lernt" eine Hierarchie der Wichtigkeit (,salience“) von Themen (,issues") aus den Medien in seine eigene "Themenhierarchie" zu übernehmen (vgl. ebd., 18). Das oben angeführte Zitat von Barry Levinson bezieht sich demnach auf dieses Übernehmen von Themensetzung („Agenda Setting“) aus den Medien in eine Themenhierarchie des Rezipienten und der damit verbundenen Ignoranz gegenüber Themen außerhalb der übernommenen „Agenda“ (Umkehrung der „Agenda Setting"-Hypothese).

Das nicht-persuasive „what to think about“ ist als Teilfolge des Agenda Setting nun nicht mehr ausreichend, denn es findet durch die Gewichtung auch eine Meinungsbeeinflussung statt (vgl. ebd., 20). Themen, die die Massenmedien auf ihre Agenda setzen, werden vom Publikum auf dessen Tagesordnung übernommen und somit als soziale Wirklichkeit empfunden (vgl. Bonfadelli 2001, 361). „Massenmedien können [...], indem sie beeinflussen, worüber wir nachdenken, zumindest in bestimmten Situationen auch beeinflussen, was wir denken." (Brettschneider 1994, 226) P. Rössler $(1997,20)$ spricht hier von einer „subtilen Persuasionstrategie“, die dem Rezipienten die „Wichtigkeit von Themen suggeriert“, die „mit bestimmten Werten besetzt sind."

Dieser medienzentrierte Ansatz ist nun eine weitere Teilfolge, diesmal jedoch mit persuasivem Charakter. Die Platzierung eines Beitrages in einer Nachrichtensendung oder auf dem Titelblatt einer Zeitung trifft bereits eine Aussage über die vermeintliche Relevanz eines Themas. Dem Rezipienten erscheinen ,alle Nachrichten, die am Anfang einer Sendung stehen, besonders bedeutungsvoll, denn die Information ist immer, so meinen die Zuschauer, die wichtigste." (ebd., 21) Behr/Iyengar konnten in einer Längsschnittuntersuchung einen substantiellen Platzierungseffekt nachweisen. In ihren Untersuchungen gewann die „Lead story“ eine besondere Bedeutung: „It is the lead story that serves as the agenda setting force." (ebd.)

Um den Sexskandal des Präsidenten als „Lead story“ zu verdrängen ist im Film $W a g$ the Dog etwas noch Skandalöseres nötig, um den besten Sendeplatz zu ergattern - ein Krieg. Diese Problemlösung entspricht einer Strategie, die W. Russell Neuman 1990 in einer von ihm vorgeschlagenen Typologie medialer Issues oder Themen als Krise bezeichnet. Neuman unterscheidet dabei vier Issues:

„1. Krisen: Krisen sind zeitlich begrenzt und besitzen Anfang, Höhepunkt und Ende.

2. Probleme: Probleme sind nicht zeitlich begrenzt, sondern andauernde Streitfragen. 3. Symbolische Krisen: Symbolische Krisen sind Probleme, die für eine kurze Zeit in Krisenstatus erhoben sind.

4. Nicht-Probleme: Nicht-Probleme sind nicht zeitlich begrenzt und nehmen in der Öffentlichkeit eine eher unbedeutende Rolle ein. " (Pöstinger 2008, 3)

In Wag the Dog wird der Krieg als Krise nach diesem dramaturgischen Muster inszeniert (vgl. Youtube-Sequenz und Sequenzplan, Seq. 8, 9).

Der Kampf um „Lead stories“ in der medialen Nachrichtenwelt ist das zentrale Thema und das Fundament der Dramaturgie des Films. Ein stetes Hin und Her zwischen „Lead stories“ des PR-Teams des Präsidenten und des PR-Teams des Widersachers Neal erinnert an zwei Werbagenturen, die sich mit Werbekampagnen bekriegen. In der „Agenda Setting“-Semiotik ist hier vom Priorities-Modell die Rede. Nach diesem Modell übernimmt der Rezipient einfach die Agenda bzw. Rangreihe der Medien. Damit ist das Priorities-Modell eindeutig das Modell, das die „stärksten“ Medien impliziert (vgl. Eichhorn 2005, 16).

Aufmachung der Berichterstattung - Präsentationsvariablen

Ähnlich dem Kompetenzbereich des ,product placement" aus der Werbung, ist das Produzententeam im Film damit beschäftigt Meldungen so zu verpacken, dass sie vom Publikum geglaubt und angenommen werden. Einen Teil des satirischen Charakters erlangt der Film durch die an Werbeagenten erinnernde Herangehensweise der Filmprodu- 
zenten an Themen der Weltpolitik. Die oben bereits angesprochene Platzierung eines Beitrags ist in den Massenmedien im „Konkurrenzkampf um die Aufmerksamkeit" (Nolte 2005, 83) der Öffentlichkeit eine der effektivsten Waffen. Es gibt aber noch andere Faktoren, die das "Agenda Setting“ so wirkungsvoll erscheinen lassen. Die „Prominenz eines Themas in den Medien“ äußert sich darüber hinaus ,in Häufigkeit, Umfang und Aufmachung der Berichterstattung dazu“" (Rössler 1997, 18). Letzteres ist neben der Platzierung des Beitrags als dramatischer Kampf um die „Lead story“ das zweite wichtige an die Theorie des „Agenda Setting“ angelehnte Thema des Filmes.

In der eigenen Semiotik des „Agenda Setting"-Ansatzes ist im Bereich der Aufmachung eines Beitrages gleich von mehreren Präsentationsvariablen die Rede: Lebendigkeit (,vividness"), Überraschung, Personalisierung, Eindeutigkeit, Negativismus, Konflikt. Alle Bereiche der Aufmachung haben eines gemeinsam, und zwar die Intention beim Rezipienten Betroffenheit zu erzeugen. McCombs spricht hier von einem dem Rezipienten innewohnenden „BetroffenheitsKontinuum" in dem er das jeweilige Thema verortet (Rössler 1997, 170).

\section{Lebendigkeit („,vividness“)}

Iyengar definierte den Begriff der Lebendigkeit „vividness“ als Charakteristikum der Aufmachung von Berichten und vermutete, dass diese Lebendigkeit positiven Einfluss hat auf die Themenstrukturierung des Rezipienten. Während Beiträge, die farblos („pallid") ,in allgemeinen Dimensionen“ (Rössler 1997, 153) aufbereitet werden, in der Wahrnehmung des Rezipienten in den Hintergrund geraten. Im Film wird der Krieg von S. Motss und seinem Produzententeam „aufgemacht“. Um den Bericht über den Krieg ,visuell eindrucksvoller und damit mit mehr ,vividness“ auszustatten, wird von ihm eine Schauspielerin mit passendem Äußerem gewählt, ihr ein Kätzchen auf den Arm gesetzt und die Geräuschkulisse der Inszenierung so gestaltet, dass ein Höchstmaß an „Lebendigkeit“ erzielt wird (vgl. YoutubeSequenz und Sequenzplan, Seq. 12, 13).

\section{Überraschung}

Die überraschende Berichterstattung von Themen mit hoher Relevanz, so lautet die Hypothese, erzeugt einen hohen „AgendaSetting-Effekt“. Diesen starken Effekt bezeichnen Schönbach/Früh $(1984,317)$ als „Injektionsnadel-Effekt“. Überraschungen werden vom Publikum als besonders interessant wahrgenommen und die Medienberichte werden deswegen aufmerksamer rezipiert. Im Film ist also der überraschende Moment der Berichterstattung vom inszenierten Krieg gerade in der Ablenkung von einem so eklatanten Zwischenfall, wie der des Sexskandals, von sehr großer Bedeutung. Des Weiteren soll die überraschende Präsentation ein kritikloses Hinnehmen der Neuigkeiten bzw. Aussagen auslösen. So verfügt der Rezipient „kaum über prästabilisierte Orientierungsmuster, welche die Reaktion auf solche Aussagen determinieren, und ist somit neuen Aussagen gegenüber relativ offen." (Rössler 1997, 156) Der Effekt wird im Film an der Szene deutlich, in der C. Brean im Fernsehen sieht, wie Neal, der Gegenspieler des Präsidenten, den Krieg für beendet erklärt und die Reaktion des Publikums simuliert (vgl. Youtube-Sequenz und Sequenzplan, Seq. 20). „Der Krieg ist aus. Ich habe es im Fernsehen gesehen."4

Wegen des Sättigungseffekts („saturation“) besteht für bereits bekannte Themen, denen kein überraschendes Moment mehr anhaftet, „nur eine geringe Chance, ihre Bedeutung weiter zu steigern“, so lautet die Hypothese weiter (vgl. Rössler 1997, S. 156). Im Film wird genau diese Problematik mit dem Sprung von der Präsentationsvariablen „Überraschung“ zur der der „Personalisierung" verdeutlicht. Als C. Brean und W. Ames die Beendigung des Krieges frustriert hinnehmen, hat S. Motss die geniale Idee die Berichterstattung vom Krieg auf eine neue Ebene zu führen, indem ein Held ins Leben gerufen wird (vgl. Youtube-Sequenz und Sequenzplan, Seq. 20,21,24).

Personalisierung

Mit der Einführung eines Helden wird die Präsentation der Berichterstattung personalisiert und bekommt somit neue Relevanz und Prominenz. Die Aufmachung der inszenierten Beiträge ändert sich nun im weiteren Verlauf des Filmes. Die anfänglich überraschende, aber abstrakte Berichterstattung weicht nun plötzlich einer personalisierten, lebendigen Heldengeschichte. Es werden Bilder von dem hinter der Front gefangenen Shoeman gezeigt. Ein Morsecode ist in seinen Pullover eingeritzt - „Hab Mut Mum“. Der Leitspruch „Hab Mut Mum“ und das von Motss und Brean initiierte Solidaritätshandeln, alte Schuhe in die Bäume zu werfen, werden in die Themenstruktur (Agenda) der Nachrichtensendungen aufgenommen (Sequenzplan, Seq. 24, 25). Der Shoeman Song wird komponiert und gesendet (vgl. Youtube-Sequenz ab Mitte des Ausschnitts). Diese Personalisierung bzw. Emotionalisierung hat im Film eine erneute erfolgreiche Umsetzung des „Agenda Setting“ zur Folge. Mit dem überwältigenden Wiederwahlergebnis von $83 \%$ wird das bestätigt. 
Rezipientenzentrierter Ansatz / Themenbetroffenheit

Der Film $W$ ag the Dog lässt einen rezipientenzentrierten Ansatz der „Agenda-SettingHypothese" außer Acht. Die Reaktion der Bevölkerung bzw. des Rezipienten auf die inszenierten Beiträge erscheint lediglich in Hochrechnungen zur Präsidentenwahl. Die Bevölkerung erscheint hier - der „Story“ dienlich - als äußerst manipulierbare Masse. So real der Film auch wirken mag, in Bezug auf den weiten Themenbereich des „Agenda Setting“" vermittelt er nur eine eindimensionale, medienzentrierte Sicht auf das Forschungsfeld. Diverse Untersuchungen hingegen ,verdeutlichen, dass Agenda Setting nicht [...] - wie ursprünglich geschehen - als medienzentriertes Konzept begriffen werden kann, sondern in transaktionaler Weise Publikumsvariablen einbezogen werden müssen (Rössler 1997, 172). Solche Publikumsvariablen, wie persönliche Dispositionen, das Orientierungsbedürfnis und interpersonale Kommunikationen innerhalb der Öffentlichkeit, werden im Film also nicht beleuchtet.

Somit erzeugen die inszenierten Berichterstattungen zum Krieg im Film stets die gewünschte Themenbetroffenheit in der filmischen Öffentlichkeit.

\section{Fazit}

Kritik an der fehlenden Medienkompetenz.

Die fehlende Beleuchtung eines rezipientenzentrierten Ansatzes hat aus Sicht der Filmemacher durchaus einen bestimmten Zweck. Der Film soll ja keine langweilige soziodemographische Längsschnittuntersuchung darstellen, sondern in erster Linie unterhalten. Darüber hinaus will der Film, was für die vorliegende Arbeit viel wichtiger ist, kritisieren.

Da die Bevölkerung im Film als leicht beeinflussbare Masse dargestellt wird, erschließt sich hieraus ein weiterer Kritikpunkt. Hier wird Kritik am eindimensionalen, trägen Medienrezipienten geübt. (Da kann man sich als Zuschauer angesprochen fühlen oder - als Ergebnis eines third-person-effect (Davidson 1983) diesen Kritikpunkt auf die Masse der anderen schieben, die einen umgeben. Die Filmemacher machen mit dieser Kritik auf ein weit verbreitetes Dilemma aufmerksam das Dilemma der fehlenden Medienkompetenz. Auf diesen Punkt möchte ich aber in diesem Beitrag nicht weiter eingehen, da er ein neues und weites Themengebiet umschließt, welches den Umfang der Arbeit sprengen würde.

„Salience“ und Fälschung

Wolfgang Eichhorn $(2005,3)$ definierte die zentrale Forschungsfrage des Agenda Setting folgendermaßen: „Führt die bevorzugte Behandlung bestimmter Themen in den Medien dazu, dass die Rezipienten diese für „wichtiger" halten als andere?" In der Agenda-Setting-Forschung geht es vornehmlich um die Untersuchung dieser Frage. Der Film Wag the Dog beantwortet diese Frage deutlich mit ,Ja“.

Ob Massenmedien durch Themensetzung die öffentliche Aufmerksamkeit für bestimmte Themen beeinflussen und darin erfolgreich sind, ihren Rezipienten vorzugeben, worüber sie nachdenken, scheint empirisch bewiesen zu sein. Viele Umfragen und Statistiken wurden hierzu unternommen. ${ }^{5}$ Alle mit dem gleichen positiven Ergebnis. Trotz dieser wissenschaftlichenpositiven Ergebnis. Trotz dieser wissenschaftlichen Ergebnissicherungen und der Tatsache, dass der „Agenda Setting“-Ansatz aus Sicht der Philosophiehistorie mit Gedankenkonstrukten diverser Philosophen vereinbar ist (man denke an das „Tabula rasa“ Prinzip von J. Locke oder den Marxschen Menschen als Produkt seiner Umwelt), ist in der weiteren Kommunikationsforschung die „Agenda Setting““These zum Teil relativiert worden.

Gerade die rezipientenzentrierten Publikumsvariablen stehen oft im Gegensatz zur medienzentrierten Theorie der „Allmacht“ der Medien. Insbesondere das Prioritätenmodell gilt heute eher als zweifelhaft. Dennoch ist es unübersehbar, dass Themenwahl und -Strukturierung einzelner Themen der Medien mit Themenprioritäten der Rezipienten zusammenhängen. Gerade bei medial ausgetragenen Wahlkämpfen, wie im Film gezeigt, scheint der „Agenda Setting“-Ansatz besonders gut zu greifen. Diese Erkenntnis hatte der Begründer der „Agenda Setting““Hypothese McCombs schon vor 36 Jahren. „While the mass media may have little influence on the direction or intensity of attitudes, it is hypothesized that the mass media set the agenda for each political campaign, influencing the salience of attitudes towards the political issues". 35 Dass diese „salience“ beeinflussbar ist und mit Fälschungen von Berichterstattungen diese Beeinflussungen fatale Folgen für die Bevölkerung haben können, zeigt der Film.

\section{Der Kampf um Aufmerksamkeit}

Die von B. Levinson angesprochene Möglichkeit, „die Aufmerksamkeit von einem nationalen Dilemma abzulenken" impliziert eine Lenkung der öffentlichen Aufmerksamkeit. Diese Lenkung der öffentlichen Aufmerksamkeit beschreibt Georg Franck (2007, 155) als eine gesellschaftliche Macht, die einer finanziellen Macht heutzutage in nichts nachsteht. „Wie die große Industrie einst ins 
Zentrum der gesellschaftlichen Macht rückte, so haben inzwischen die Massenmedien diese Stellung erobert.“ Die Ausübung dieser gesellschaftlichen Macht erfolgt über die Massenmedien und da zum Teil über das hier besprochene Phänomen des „Agenda Setting“" Die beeinflussende Kraft der medialen Themenstrukturierung auf den Rezipienten bezieht sich bei Franck auf die begrenzte Ressource Aufmerksamkeit ,in den Grenzen der mit dem Wachen und Schlafen wechselnden Präsenz" (ebd., 49).

Aufmerksamkeit auf sich zu ziehen, ist jedoch nur eine Sache, wie man sich präsentiert, die andere. Schließlich bezieht sich die Wirkung des „Agenda Setting“ nicht nur auf das "what to think about", sondern auch auf das ,how to think about". Der Film Wag the Dog beschäftigt sich, wie in der vorliegenden Arbeit erläutert, mit der Wirkungstheorie McCombs'. Zum einen zeigt er den dramatischen Kampf um die beste Platzierung eines Themas („lead story“) und zum anderen die unterhaltende Inszenierung bzw. „Aufmachung" der Themen um die Aufmerksamkeit der Menschen zu erheischen und zu lenken. Der Kampf um die knappe Ressource Aufmerksamkeit wird, so Franck (2007, 113ff.), auch in der Realität von den großen Medienkonzernen und der Politik unerbittlich betrieben. Im Film kostet die Gier nach dieser Aufmerksamkeit Stanley Motss sogar das Leben.

\section{Anmerkungen}

1 Das Buch American Hero von Larry Beinhart diente den Filmemachern von $W$ ag the Dog als Vorlage.

2 Budd Schulberg, Drehbuchautor und Screenwriter des Filmes „A face in the crowd", zitiert aus dem dokumentarischen Hintergrundmaterial „Von Washington nach Hollywood“ der DVD Wag the Dog erschienen bei Concorde Home Entertainment, produziert von Mark Rance; Min. 4, Sek. 48.

3 Barry Levinson, Regisseur und Produzent des Filmes $W$ ag the Dog, zitiert aus dem dokumentarischen Hintergrundmaterial „Von Washington nach Hollywood“ der DVD Wag the Dog erschienen bei Concorde Home Entertainment, produziert von Mark Rance; Min. 23, Sek. 30.

4 C. Brean (Robert de Niro) in Wag the Dog, 1998; Min 45, Sek. 36.

5 Siehe Grafiken in URL: http://www. agendasetting.com/res_theory.php [18.4.2008]

6 McCombs/Shaw zit. nach Rössler (1997, 16)

\section{Literatur}

Beinhart, Larry (2003): American Hero: Wag the Dog. Köln.

Bonfadelli, Heinz (2001): Medienwirkungsforschung, Einführung in die Publizistikwissenschaft. Bern/Wien.

Brettschneider, Frank (1994): Agenda Setting. Forschungsstand und politische Konsequenzen. In: Jäckel, Michael/WinterhoffSpurk, Peter (Hrsg.): Politik und Medien. Analysen zur Entwicklung der politischen Kommunikation, Berlin, S. 211-229.

Davison, W. Phillips (1983): The thirdperson effect in communication. In: Public Opinion Quarterly, 47, S. 1-15.

Eichhorn, Wolfgang (2005): Agenda-SettingProzesse: eine theoretische Analyse individueller und gesellschaftlicher Themenstrukturierung (Erstveröffentlichung: Reihe Kommunikationswissenschaftliche Studien, Band 16, Verlag Reinhard Fischer, München, 1996); URL: http://epub.ub.unimuenchen.de/734/1/Agenda SettingProzesse.pdf [Zugriff: 15.09.08].

Franck, Georg (2007): Ökonomie der Aufmerksamkeit - Ein Entwurf. München.

Nolte, Kristina (2008): Der Kampf um Aufmerksamkeit: Wie Medien, Wirtschaft und Politik um eine knappe Ressource ringen. Franfurt a. M.

Pöstinger, Markus (2008): Agenda-Setting Ansätze und Theorien. URL: http://www. datamoon.de/misc/Seminar AgendaSetting.pdf [Zugriff: 20.9.2008].

Rath, Matthias (2000): Medienwirkungsforschung in Deutschland - eine Annäherung. In: Buchwissenschaft und Buchwirkungsforschung (VIII. Leipziger Hochschultage für Medien und Kommunikation) Hrsg. v. Dietrich Kerlen/Inka Kirste. Leipzig, S. 89-98.

Rath, Matthias (2006): Wahrhaftigkeit des Journalismus: Moralanspruch oder Marktfaktor?; In: Medienkritik heute. Grundlagen, Beispiele und Praxisfelder. Hrsg. v. Horst Niesyto/ Matthias Rath/Hubert Sowa. München, S. 117-128.

Rössler, Patrick (1997): Agenda-Setting. Theoretische Annahmen und empirische Evidenzen einer Medienwirkungshypothese. Opladen.

Schönbach, Klaus/Früh, Werner (1984): Der dynamisch-transaktionale Ansatz II: Konsequenzen. In: Rundfunk und Fernsehen (32), S. 315-329.

Wag the Dog (1998): Produktion: New Line Ginema / Tribeca Productions / Baltimore Pictures; Regie: Barry Levinson; Drehort/ zeit: USA, 1997; deutscher Titel: Wag the Dog - Wenn der Schwanz mit dem Hund wedelt, DVD erschienen bei Concorde Home Entertainment 2003. 


\section{Sequenzplan zu Barry Levinson: Wag the Dog (1998)}

\section{PETER SCHMITT}

(Kursiv geschrieben: Ein Versuch eine an V. Propps „Morphologie des Märchens“ (1975) angelehnte Figureneinteilung, die sich auch für Filme eignet, zu integrieren und den Film dramaturgisch in einen Dreiakter einzuteilen.)

\begin{tabular}{|c|c|c|c|}
\hline Nr. & Titel & Inhalt & Besonderheit \\
\hline 1 & Vorspann & $\begin{array}{l}\text { Nach Vorstellung von Produktionsfirma und } \\
\text { Hauptproduzenten werden folgende Sätze } \\
\text { nacheinander eingeblendet: } \\
\text { "Warum wedelt ein Hund mit seinem } \\
\text { Schwanz?” } \\
\text { „Weil ein Hund schlauer ist als sein } \\
\text { Schwanz.“ } \\
\text { „Wenn der Schwanz schlauer wäre, würde der } \\
\text { Schwanz mit dem Hund wedeln.“" }\end{array}$ & $\begin{array}{l}\text { Unterbrechung des } \\
\text { Vorspanns; } \\
\text { Schrift; } \\
\text { Leitmotiv: Musik im } \\
\text { Off }\end{array}$ \\
\hline 2 & Werbetrailer & $\begin{array}{l}\text { Werbetrailer für einen amtierenden Präsiden- } \\
\text { ten }\end{array}$ & $\begin{array}{l}\text { Leitmotiv: Werbetrai- } \\
\text { ler für den Präsidenten } \\
\text { - „Wechsle niemals } \\
\text { die Pferde auf halber } \\
\text { Strecke.“ } \\
\text { Unterbrechung des } \\
\text { Vorspanns; } \\
\text { TV im Film }\end{array}$ \\
\hline 3 & $\begin{array}{l}\text { Vorspann Fortset- } \\
\text { zung / „Mr. Alles- } \\
\text { kleber“" }\end{array}$ & $\begin{array}{l}\text { Ankündigung der Schauspieler; } \\
\text { Einführung des Helden - 1. Akt: } \\
\text { Conrad Brean (Robert de Niro) betritt das } \\
\text { Weiße Haus und wird in ein Hinterzimmer } \\
\text { geführt - Treppen, vertrackte Gänge. } \\
\text { „Das ist er. Das ist Mr. Alleskleber.“ }\end{array}$ & $\begin{array}{l}\text { Vorspann mit Hand- } \\
\text { lung; } \\
\text { Leitmotiv: Das Weiße } \\
\text { Haus } \\
\text { Überwachungskamera }\end{array}$ \\
\hline 4 & $\begin{array}{l}\text { Konspirative Run- } \\
\text { de }\end{array}$ & $\begin{array}{l}\text { Es ist Abend. Der Beraterstab des Präsidenten } \\
\text { (des Senders) befindet sich in einer Krisensit- } \\
\text { zung in einem Kellerraum des Weißen Hau- } \\
\text { ses. Brean weist die Berater darauf hin, dass } \\
\text { Geheimhaltung oberste Priorität hat. Er gibt } \\
\text { Anweisungen. Er ist der Chef der Runde. } \\
\text { Grund der Krise: Der Präsident hat sich an } \\
\text { einem Schulkind vergangen. Das Schulmäd- } \\
\text { chen hat sich bereits an die Presse gewandt, } \\
\text { die Nachricht wird am nächsten Tag auf der } \\
\text { Titelseite der „Washington Post“ erscheinen. } \\
\text { Diese Katastrophe gilt es nun abzuwenden. } \\
\text { Conrad Brean ist hierfür der Experte. Er leitet } \\
\text { Ablenkungsmanöver ein. } \\
\rightarrow \text { Ablenkung durch Lügen: Der Präsident ist } \\
\text { krank; Der B3 Bomber }\end{array}$ & $\begin{array}{l}\text { Nahaufnahmen } \\
\text { Leitmotiv: Brean strei- } \\
\text { tet mit Pressespreche- } \\
\text { rin }\end{array}$ \\
\hline 5 & $\begin{array}{l}\text { Werbetrailer des } \\
\text { Konkurrenten }\end{array}$ & $\begin{array}{l}\text { Die Runde sieht sich den Werbetrailer für den } \\
\text { Präsidentschaftskandidaten der Gegenseite an. } \\
\text { Der Trailer ist vernichtend, dementsprechend } \\
\text { die Reaktionen. } \\
\text { Conrad Brean spielt kurz, laut denkend, die } \\
\text { Pressekonferenz vom nächsten Tag durch: } \\
\text { B3-Bomber Einsatz! - B3-Bomber? Wegen } \\
\text { der Krise! Welche Krise? } \\
\text { Na, daran arbeite ich. }\end{array}$ & $\begin{array}{l}\text { Der Trailer ist mit } \\
\text { Musik unterlegt. } \\
\text { In ihr heißt es: „Dark } \\
\text { heaven for little girls“; } \\
\text { TV im Film }\end{array}$ \\
\hline
\end{tabular}




\begin{tabular}{|c|c|c|c|}
\hline 6 & Flugzeug & $\begin{array}{l}\text { Gespräch im Flüsterton zwischen Conrad } \\
\text { Brean und seiner Assistentin - der ersten Helferin } \\
\text { - Winifred Ames (- Anne Heche) über Insze- } \\
\text { nierung eines Krieges gegen Albanien. } \\
\text { Brean: „Wer kennt schon Albanien.“ } \\
\text { Ames ist skeptisch. Brean erzählt ihr von Me- } \\
\text { dieninszenierungen und Ablenkungen in der } \\
\text { Vergangenheit. } \\
\text { Brean: „Wir müssen ablenken, wir müssen sie } \\
\text { einfach nur ablenken. Wir haben nicht mal } \\
2 \text { Wochen bis zur Wahl“ } \\
\text { Ames: „Was kann die noch retten?! Was kann } \\
\text { die noch retten?!“ } \\
\text { Brean: „Na, daran arbeite ich!“ }\end{array}$ & $\begin{array}{l}\text { Flüsterton nachts im } \\
\text { Flugzeug - Intimität }\end{array}$ \\
\hline 7 & Am Flughafen & $\begin{array}{l}\text { Nachrichten im Fernsehen } \rightarrow \text { Der Präsident } \\
\text { als Kinderschänder; } \\
\text { Der Gegenspieler: Präsidentschaftskandidat Neal } \\
\text { fordert den Präsidenten im Fernsehen auf, } \\
\text { zurückzutreten. } \\
\text { Lange Einstellung: } \\
\text { Brean wird von hinten gezeigt, wie er durchs } \\
\text { Gate läuft, während Ames eifrig telefoniert und } \\
\text { seine Anweisungen ausführt. (- Hinweis auf } \\
\text { Hierarchie) }\end{array}$ & $\begin{array}{l}\text { Überwachungskamera } \\
\text { am Flughafen }\end{array}$ \\
\hline 8 & $\begin{array}{l}\text { In der Villa des } \\
\text { Filmproduzenten }\end{array}$ & $\begin{array}{l}\text { Brean und Ames betreten die Villa. } \\
\text { Einführung des zweiten Helfers: Stanley Motss } \\
\text { (- Dustin Hoffman) } \\
\text { Er liegt im privaten Solarium. Der Raum leuch- } \\
\text { tet blau. Motss zu seinem Bediensteten: „Ach, } \\
\text { und bringen sie mir meinen Gemüseshake Ra- } \\
\text { mon. Und erinnern sie mich daran mich in } 10 \\
\text { Min. umzudrehen, um die andere Seite zu } \\
\text { bräunen.“ } \\
\text { Brean und Ames stehen verlegen im blauen } \\
\text { Licht und warten empfangen zu werden. } \\
\text { Es folgt ein Gespräch mit dem Produzenten } \\
\text { über Hinhaltetaktik. } \\
\text { Sie sehen sich gemeinsam die Pressekonferenz } \\
\text { im Weißen Haus an. } \\
\text { C. Brean stellt sich durch Telefonanruf in die } \\
\text { Pressekonferenz als Hauptverantwortlicher vor } \\
\text { und demonstriert seine Macht. } \\
\text { „Was soll er sagen?“ } \\
\text { Im weiteren Verlauf des Gesprächs wird klar, } \\
\text { C. Brean will einen Krieg vom Produzenten. } \\
\text { Motss ist zunächst abgeneigt. } \\
\text { Der hält sich für genial und regt sich über die } \\
\text { Tatsache auf, dass es für Produzenten keine } \\
\text { Oskars gibt. Er bekommt zu wenig Anerken- } \\
\text { nung. }\end{array}$ & $\begin{array}{l}\text { Schuss - Gegenschuss } \\
\text { - Verfahren bei Dialo- } \\
\text { gen } \\
\text { Telefonanruf in die } \\
\text { Pressekonferenz }\end{array}$ \\
\hline
\end{tabular}




\begin{tabular}{|c|c|c|c|}
\hline 9 & $\begin{array}{l}\text { In der Villa des } \\
\text { Filmproduzenten } 2\end{array}$ & $\begin{array}{l}\text { Während Brean Motss erklärt, dass Krieg } \\
\text { Showbusiness ist und im Gedächtnis der Men- } \\
\text { schen Kriege als Motive hängen bleiben, wer- } \\
\text { den originale Fotos der Weltpolitik als solche } \\
\text { Motive eingeblendet. Durch solche Motive } \\
\text { wurden die Menschen schon immer vom ei- } \\
\text { gentlichen politischen Geschehen abgelenkt. } \\
\text { Brean: „Die eine clevere Bombe, die in einen } \\
\text { Schornstein fällt.“ (- Golfkrieg) } \\
\text { „Wer hat Kennedy getötet? Erst wurde von } \\
\text { der Presse ein betrunkener Passant angege- } \\
\text { ben!“ } \\
\text { Motss lässt sich überzeugen und ist sofort in } \\
\text { seinem Element, er denkt laut über den Auf- } \\
\text { hänger des zu inszenierenden Krieges nach. } \\
\text { Er trommelt seine Leute zusammen. } \\
\rightarrow \text { John Dean - Songwriter; Liz Buzky - Zu- } \\
\text { ständig für Leitmotive; Knüllerkönig (Denis } \\
\text { Leary) } \\
\text { In seine Überlegungen bindet er Ames und } \\
\text { Brean ein. Brean fordert eine hollywoodartige } \\
\text { Inszenierung eines Krieges. Motss: Albanische } \\
\text { Terroristen wollen eine Kofferbombe von } \\
\text { Kanada in die USA schaffen! } \\
\text { Er erklärt an einem großen Globus seine Idee. } \\
\text { Motss weist im Eifer seiner Ausführungen } \\
\text { einen Anruf des Präsidenten zurück } \rightarrow \text { Grö- } \\
\text { Benwahn }\end{array}$ & $\begin{array}{l}\text { Schuss - Gegenschuss: } \\
\text { Brean wird von unten } \\
\text { gefilmt, Motss von } \\
\text { oben } \rightarrow \text { Mächtever- } \\
\text { hältnis } \\
\\
\text { Bildkomposition: } \\
\text { Brean und Motss im } \\
\text { oberen Drittel des Bil- } \\
\text { des zu sehen. Sie ste- } \\
\text { hen hinter dem Globus } \\
\text { (unten, groß im Vor- } \\
\text { dergrund) } \\
\text { Sie werden von unten } \\
\text { gefilmt } \\
\rightarrow \text { sie spielen Gott! }\end{array}$ \\
\hline 10 & $\begin{array}{l}\text { In der Produzen- } \\
\text { tenvilla; Der Krieg } \\
\text { wird vorbereitet }\end{array}$ & $\begin{array}{l}\text { Es ist Nacht. Die kreative Runde ist komplett. } \\
\text { Die Vorbereitungen zu einem Trailer zum } \\
\text { Krieg werden durch schlechte Presse für den } \\
\text { Präsidenten unterbrochen. } \\
\text { Details werden besprochen, eine Story wird } \\
\text { erfunden: Junges albanisches Mädchen flieht } \\
\text { aus ihrem Dorf mit einem Kätzchen auf dem } \\
\text { Arm. Motss: „O.k., schreib das mit: Junges } \\
\text { Mädchen rennt aus Dorf auf Kamera zu, kör- } \\
\text { nig, Handkamera, na ja, wie in den Nachrich- } \\
\text { ten eben.“ } \\
\text { Motss: „Sie ist es, zu deren Verteidigung wir } \\
\text { mobil machen!“‘ } \\
\text { J. Dean ist dabei einen Song zum Krieg zu } \\
\text { schreiben. }\end{array}$ & $\begin{array}{l}\text { TV-Nachrichten im } \\
\text { Film } \\
\text { Nachrichtensprecher } \\
\text { im Off }\end{array}$ \\
\hline 11 & Erste Resultate & $\begin{array}{l}\text { Fahrt von Villa ins Filmstudio. } \\
\text { Krieg auf den Titelseiten der Zeitungen. } \\
\text { TV-, Radio-Nachrichten berichten vom Krieg. }\end{array}$ & $\begin{array}{l}\text { TV im Film; } \\
\text { Leitmotiv Musik; } \\
\text { Radionachrichten im } \\
\text { Off }\end{array}$ \\
\hline 12 & $\begin{array}{l}\text { Der Trailer zum } \\
\text { Krieg wird insze- } \\
\text { niert; } \\
\text { Im Bluescreen- } \\
\text { Raum }\end{array}$ & $\begin{array}{l}\text { Das Produktionsstudio ist ein großer Blue- } \\
\text { screen-Raum. } \\
\text { Im Produktionsstudio wird der Schauspielerin, } \\
\text { die das albanische Mädchen spielt Instruktio- } \\
\text { nen gegeben. Sie wird von der Assistentin zur } \\
\text { Unterzeichnung eines Schweigegelübdes über- } \\
\text { redet. } \\
\text { Das passende Kätzchen, das dem Mädchen auf } \\
\text { dem Arm sitzen soll, wird ausgesucht. } \\
\text { Ames: Der Präsident will ein Kätzchen.“ } \\
\text { Motss: „Nein, ich will ein Kätzchen!“ }\end{array}$ & $\begin{array}{l}\text { Filmproduktion im } \\
\text { Film; } \\
\text { Offen Bildform: Ka- } \\
\text { mera schwenkt schein- } \\
\text { bar unwillkürlich von } \\
\text { einer Person zur } \\
\text { nächsten } \rightarrow \text { Ge- } \\
\text { sprächsfetzen }\end{array}$ \\
\hline
\end{tabular}




\begin{tabular}{|c|c|c|c|}
\hline 13 & $\begin{array}{l}\text { Der Trailer zum } \\
\text { Krieg wird insze- } \\
\text { niert; } \\
\text { Im Schneideraum }\end{array}$ & $\begin{array}{l}\text { Der Trailer wird digital bearbeitet, das Dorf wird } \\
\text { in den bestehenden Film eingesetzt. Geräusche, } \\
\text { Schreie, Sirenen werden eingefügt. } \\
\text { Ames: „Der Präsident will ein weißes Kätz- } \\
\text { chen.“ } \\
\text { Motss: „Ich hasse es, wenn sie einem reinreden.“ } \\
\text { Parallel zu den Szenen im Schneideraum wird } \\
\text { der Gegenspieler Senator Neal im Fernsehen } \\
\text { eingeblendet, der versucht den Präsidenten zu } \\
\text { diskreditieren. } \\
\text { Brean: „Egal was Senator Neal hat, wir haben } \\
\text { einen Krieg!“ }\end{array}$ & $\begin{array}{l}\rightarrow \text { an dieser Stelle wird } \\
\text { ein Hund eingeblendet, } \\
\text { der mit dem Schwanz } \\
\text { wedelt. }\end{array}$ \\
\hline 14 & $\begin{array}{l}\text { Der Trailer wird } \\
\text { gesendet }\end{array}$ & $\begin{array}{l}\text { Im Fernseher des Filmstudios sehen sie ihren } \\
\text { Trailer in den Nachrichten. Nachrichtenspre- } \\
\text { cher: „Amerika ist nur selten Zeuge eines so } \\
\text { bewegenden Bildes der Menschheit geworden.“ } \\
\text { Positive Reaktionen von Brean, Ames und } \\
\text { Motss - Schulterklopfen. }\end{array}$ & TV im Film \\
\hline 15 & $\begin{array}{l}\text { In der Stretchlimou- } \\
\text { sine }\end{array}$ & $\begin{array}{l}\text { Brean übermittelt per Telefon seine Vorstellun- } \\
\text { gen von einer weiteren Emotionalisierung der } \\
\text { Inszenierung ans Produktionsstudio. } \\
\text { Während Brean den weiteren Verlauf der Insze- } \\
\text { nierung plant, wird die Limousine plötzlich von } \\
\text { der CIA angehalten. }\end{array}$ & Leitmotiv: Musik \\
\hline 16 & $\begin{array}{l}\text { Diskussion mit der } \\
\text { CIA }\end{array}$ & $\begin{array}{l}\text { In einer Bar werden Brean und seine Assistentin } \\
\text { zunächst von dem Chef der CIA beschuldigt, zu } \\
\text { lügen. Brean versteht es jedoch rhetorisch ge- } \\
\text { schickt das Mächteverhältnis zwischen Regie- } \\
\text { rung und CIA zu klären um sich und seine As- } \\
\text { sistentin aus dem Schlammassel rauszureden. Er } \\
\text { droht dem CIA-Chef indirekt mit einem Raus- } \\
\text { wurf. }\end{array}$ & $\begin{array}{l}\text { TV im Film - Die Se- } \\
\text { quenz ist mit Szenen } \\
\text { aus TV-Nachrichten- } \\
\text { sendungen durchsetzt. }\end{array}$ \\
\hline 17 & $\begin{array}{l}\text { Zurück in der Li- } \\
\text { mousine }\end{array}$ & $\begin{array}{l}\text { Ames: „Brean, Sie könnten einem Hund einen } \\
\text { Fleischlaster ausreden.“ }\end{array}$ & \\
\hline 18 & $\begin{array}{l}\text { Der Krieg wird zur } \\
\text { Wirklichkeit }\end{array}$ & $\begin{array}{l}\text { Nachrichtensender senden weitere berührende } \\
\text { Szenen aus dem vermeintlichen Kriegsgesche- } \\
\text { hen. Der Präsident überreicht seinen Mantel an } \\
\text { eine alte Frau und wird als Held empfunden. } \\
\text { Der fertige Song zum Krieg ist zu hören. }\end{array}$ & $\begin{array}{l}\text { TV im Film; } \\
\text { Musik im Off }\end{array}$ \\
\hline 19 & $\begin{array}{l}\text { Die Produktion des } \\
\text { Songs; } \\
\text { Im Tonstudio } 1\end{array}$ & $\begin{array}{l}\text { Mehrere Sänger sind beim Einsingen des patrio- } \\
\text { tischen Liedes zu sehen. } \\
\text { Parallel zu der Songproduktion findet sich Brean } \\
\text { im Tonstudio ein. }\end{array}$ & $\rightarrow$ Musik im On \\
\hline 20 & $\begin{array}{l}\text { Neal beendet den } \\
\text { Krieg; } \\
\text { Im Tonstudio } 2\end{array}$ & $\begin{array}{l}\text { Im Fernsehen beendet der Präsidentschaftskan- } \\
\text { didat Neal offiziell den Krieg. Er untermauert } \\
\text { seine Behauptungen mit der CIA als Quelle. Für } \\
\text { Brean bricht eine Welt zusammen. } \\
\text { Brean: „Der Krieg ist vorbei, ich habe es im } \\
\text { Fernsehen gesehen.“ } \\
\text { Motss: „Er kann den Krieg nicht beenden! Er ist } \\
\text { doch nicht der Produzent!“ } \\
\text { Motss ist guter Dinge und wähnt sich bereits in } \\
\text { der Erschaffung einer Heldengeschichte. Für ihn } \\
\text { ist es ein Glück, dass der Krieg zu Ende ist, } \\
\text { denn nun ist Platz für einen zweiten Akt. }\end{array}$ & $\begin{array}{l}\text { Wackelige Handkamera; } \\
\text { Amateurhafter Zoom } \\
\rightarrow \text { Musik im Off }\end{array}$ \\
\hline
\end{tabular}




\begin{tabular}{|c|c|c|c|}
\hline 21 & Der gute alte Schuh & $\begin{array}{l}\text { Der 2. Akt: Motss hat gleich die Idee von einem } \\
\text { alten Schuh. Der passende Song wird dazu ge- } \\
\text { schrieben. Während der neue Song zu hören ist, } \\
\text { denken sich Motts und der Knüllerkönig eine } \\
\text { Rede für den Präsidenten aus, in der die Be- } \\
\text { kanntmachung eines in Albanien zurückgelasse- } \\
\text { nen amerikanischen Helden stattfinden soll. Der } \\
\text { Held soll Schoeman heißen. } \\
\text { Brean: „Ist es möglich, das es ein bisschen ver- } \\
\text { kratzter klingt, mit Rauschen?“ }\end{array}$ & $\begin{array}{l}\text { Einblendung eines Plat- } \\
\text { tenspielers mit der Plat- } \\
\text { te „Good old shoe“ am } \\
\text { Ende der Sequenz; } \\
\text { Musik erst im On, dann } \\
\text { im Off }\end{array}$ \\
\hline 22 & $\begin{array}{l}\text { Zurück im Weißen } \\
\text { Haus }\end{array}$ & $\begin{array}{l}\text { Die Präsidentenrede wird eingereicht. } \\
\text { Der Beraterstab des Präsidenten hat sich nun } \\
\text { um den Hollywoodproduzenten Motss erwei- } \\
\text { tert. Das weitere Vorgehen wird geplant. } \\
\text { Der Trailer der Gegenseite ist zu sehen. } \\
\text { Motss versucht die schlechte Stimmung inner- } \\
\text { halb der Mitarbeiter zu verbessern. } \\
\text { Brean telefoniert mit dem Präsidenten und muss } \\
\text { mit Erschütterung feststellen, dass der Präsident } \\
\text { die vorbereitete Rede nicht halten will. Motss ist } \\
\text { empört und möchte unbedingt den Präsidenten } \\
\text { sprechen. }\end{array}$ & $\begin{array}{l}\text { Leitmotiv: Das Weiße } \\
\text { Haus; } \\
\text { TV im Film } \\
\text { Leitmotiv: Brean strei- } \\
\text { tet mit Pressesprecherin }\end{array}$ \\
\hline 23 & Motss als Präsident & $\begin{array}{l}\text { Motss ist hinter dem Schreibtisch des Präsiden- } \\
\text { ten zu sehen. Er hält die Rede so, wie er sich das } \\
\text { vorstellt, und erklärt den emotionalen Moment } \\
\text { der Ansprache. Er überzeugt den Präsidenten. } \\
\text { Brean ist begeistert: „Sie haben es wieder einge- } \\
\text { renkt!“" } \\
\text { Brean: „Es ist, wie Plato es einmal gesagt hat, } \\
\text { scheißegal wie man dahin gelangt, solange man } \\
\text { dahin gelangt.“ }\end{array}$ & \\
\hline 24 & $\begin{array}{l}\text { Der Präsident hält } \\
\text { die Rede im TV }\end{array}$ & $\begin{array}{l}\text { Die Nation lernt nun den vermeintlichen Solda- } \\
\text { ten Shoeman kennen. Er trägt die Nummer } 303 . \\
\text { Er soll sich noch hinter der feindlichen Linie in } \\
\text { Albanien befinden. } \\
\text { Ein Foto wird gezeigt, auf dem der Soldat einen } \\
\text { zerschlissenen Pullover an hat. In den Pullover } \\
\text { ist in Morsecode eine Botschaft zu sehen: „Hab } \\
\text { Mut Mum.“ }\end{array}$ & $\begin{array}{l}\text { TV im Film; } \\
\text { Leitmotiv: Das Weiße } \\
\text { Haus; } \\
\text { Foto im Film }\end{array}$ \\
\hline 25 & Alte Schuhe & $\begin{array}{l}\text { Brean und Motss schmeißen alte Schuhpaare auf } \\
\text { einen Baum. Die Nachricht verbreitet sich } \\
\text { schnell. Bald hängen in ganz Amerika die Schu- } \\
\text { he auf Bäumen, Straßenlaternen und Stromka- } \\
\text { beln. Der (alte) neue Schuhsong wird im Radio } \\
\text { gespielt. James Belushi, der sich selbst spielt und } \\
\text { scheinbar Albaner ist, hält eine Rede auf alba- } \\
\text { nisch. Ein Denkmal für Shoeman soll errichtet } \\
\text { werden. Der Shoeburger soll bei Burger King } \\
\text { rausgebracht werden. T-Shirts mit „holt ihn } \\
\text { zurück“ werden gedruckt. }\end{array}$ & $\begin{array}{l}\text { TV im Film; } \\
\text { Einspielungen einer } \\
\text { Tafel mit dem Count- } \\
\text { down zur Wahl: } 8,7,6 \\
\text { Tage noch; } \\
\text { Leitmotive: Brean strei- } \\
\text { tet mit Pressespreche- } \\
\text { rin; Das Weiße Haus }\end{array}$ \\
\hline 26 & In der Limousine & $\begin{array}{l}\text { Im Fernseher der Limousine ist der Wahltrailer } \\
\text { für den Präsidenten zu sehen. Weitere Pläne } \\
\text { werden geschmiedet. Motss ist traurig, weil nach } \\
\text { der ganzen Sache niemand weiß, wer sie veran- } \\
\text { staltet hat. } \\
\text { Der Song „Have courage mum“ ist zu hören. }\end{array}$ & $\begin{array}{l}\text { TV im Film; } \\
\text { Leitmotiv: Wahltrailer: } \\
\text { Wechsle nicht die Pfer- } \\
\text { de auf halber Strecke }\end{array}$ \\
\hline
\end{tabular}




\begin{tabular}{|c|c|c|c|}
\hline 27 & Im Privatjet & $\begin{array}{l}\text { Brean, seine Assistentin und Motss sitzen im } \\
\text { Privatjet. Sie rekapitulieren, diskutieren und phi- } \\
\text { losophieren. } \\
\text { Für Motss ist der Song der Höhepunkt seiner } \\
\text { Arbeit. Im Flugzeug sieht man im Fernsehen ein } \\
\text { Konzert der Band, die den Song „Have courage } \\
\text { mum“ spielt. }\end{array}$ & TV im Film \\
\hline 28 & $\begin{array}{l}\text { Am Gefängnisflug- } \\
\text { hafen }\end{array}$ & $\begin{array}{l}\text { Die gesuchte Person: Der Schwerverbrecher Shoe- } \\
\text { man wird von Soldaten in den Privatjet geführt. } \\
\text { Die Assistentin gerät in Unruhe, während Brean } \\
\text { und Motss entzückt sind. Brean telefoniert mit } \\
\text { Boston, wo Shoeman ein großer Empfang berei- } \\
\text { tet wird. Er plant dort folgendes Schauspiel: Ein } \\
\text { Junge soll die Absperrungen durchbrechen und } \\
\text { läuft zu Old Shoe (so wird der vermeintliche } \\
\text { Kriegsheld oft genannt). Brean: „Ein kleiner } \\
\text { Hund kommt angelaufen, er liebt Shoeman.“ } \\
\text { Ames, Motss und Brean lesen die Gefangenen- } \\
\text { akte: } \\
\text { Shoeman hat eine Nonne vergewaltigt. Er ist, } \\
\text { wenn er seine Medikamente bekommt, ruhig. } \\
\text { Brean entschließt sich nun, doch wieder nach } \\
\text { Washington zu fliegen. Trotz Unwetterwarnun- } \\
\text { gen starten sie. }\end{array}$ & Leitmotiv: Musik \\
\hline 29 & Während des Fluges & $\begin{array}{l}\text { Das Flugzeug gerät in Turbulenzen. Der Um- } \\
\text { gang mit dem Gefangenen ist heikel. Die Situati- } \\
\text { on eskaliert. } \\
\text { Das Flugzeug stürzt ab. Alle Insassen überleben. }\end{array}$ & Schnelle Schnitte \\
\hline 30 & Im Weißen Haus & Der Beraterstab wartet auf Lebenszeichen. & $\begin{array}{l}\text { Leitmotive: Musik; Das } \\
\text { Weiße Haus; } \\
\text { TV im Film }\end{array}$ \\
\hline 31 & $\begin{array}{l}\text { An der Absturz- } \\
\text { stelle }\end{array}$ & $\begin{array}{l}\text { Streit zwischen Ames und Motss. } \\
\text { In einem Handfernseher, den sie in den Trüm- } \\
\text { mern der Maschine finden, ist eine Rede des } \\
\text { Senators Neal zu sehen. }\end{array}$ & TV im Film \\
\hline 32 & Im Mähdrescher & $\begin{array}{l}\text { Sie trampen mit einem Mähdrescher nach Wa- } \\
\text { shington. Sie diskutieren die Lage. Der Gefange- } \\
\text { ne ist einem äußerst verwirrten Zustand und } \\
\text { scheint für Brean nicht der Richtige zu sein. } \\
\text { Motss muss ihn überzeugen, dass Shoeman der } \\
\text { Richtige ist. } \\
\text { Diskussion um Geheimhaltung. Motss will An- } \\
\text { erkennung für seine taten als Produzent des } \\
\text { Krieges, Brean mahnt ihn zur Geheimhaltung. }\end{array}$ & $\begin{array}{l}\text { Licht: } \\
\text { Starkes weißes Licht } \\
\text { beleuchtet die Insassen } \\
\text { von hinten }\end{array}$ \\
\hline
\end{tabular}




\begin{tabular}{|c|c|c|c|}
\hline 33 & An der Raststätte & $\begin{array}{l}\text { Sie machen an einer Raststätte halt. Sie sehen } \\
\text { dort im Fernsehen, wie der Konkurrent des } \\
\text { Präsidenten die Regierung dazu auffordert, } \\
\text { Shoeman zu zeigen, weil er es sonst nicht zögert } \\
\text { zu sagen, ,,reihen sie ihn ein in eine ganze Serie } \\
\text { von gebrochenen Versprechen.“ } \\
\text { Brean ruft in Washington an und gibt durch, } \\
\text { dass Shoeman in Sicherheit an einem geheimen } \\
\text { Ort aufbewahrt wird. Er hätte leichte Verletzun- } \\
\text { gen und braucht einen Tag Zeit um sich zu er- } \\
\text { holen. Daraufhin sehen sie eine Pressekonfe- } \\
\text { renz im Weißen Haus, in welcher der Sprecher } \\
\text { das weitergibt, was Brean telefonisch durchge- } \\
\text { geben hat. } \\
\text { Während sie vor dem Fernseher stehen, macht } \\
\text { sich der Gefangene Shoeman an eine Frau ran, } \\
\text { die an der Raststätte wohnt. } \\
\text { Er wird vom Vater bzw. Tankwart erschossen. } \\
\text { Brean und seine Assistentin sind am Boden } \\
\text { zerstört, während Motss unermüdlich positiv } \\
\text { bleibt und den Vorfall als Glücksfall darstellt. } \\
\text { Er sagt: „Und was gibt es Besseres als die trium- } \\
\text { phale Heimkehr eines Kriegshelden?“ } \\
\rightarrow \text { Schnitt zur nächsten Sequenz }\end{array}$ & $\begin{array}{l}\text { Roadmovie-ähnlich; } \\
\text { TV im Film }\end{array}$ \\
\hline 34 & Rückkehr im Sarg & $\begin{array}{l}\text { Der feierliche Empfang des Sarges wird mit } \\
\text { andächtigem Kriegschor musikalisch untermalt. } \\
\text { Ein Hund rennt hinter dem Sarg her. }\end{array}$ & $\begin{array}{l}\text { Musik erst im Off, } \\
\text { dann im On }\end{array}$ \\
\hline 35 & In einem Büroraum & $\begin{array}{l}\text { Der 3. Akt: } \\
\text { Brean und Motss loben sich gegenseitig und } \\
\text { klopfen sich auf die Schulter zur erfolgreichen } \\
\text { Mission, während sie das Begräbnis im Fernse- } \\
\text { hen anschauen. Doch dann sehen sie im TV } \\
\text { eine Diskussionsrunde zum Thema Präsident- } \\
\text { schaft. } \\
\text { Diese TV-Sendung entfacht eine hitzige Diskus- } \\
\text { sion zwischen Brean und Motss. } \\
\text { Motss will Anerkennung. } \\
\text { Er verlässt den Raum mit der Absicht sich seine } \\
\text { Anerkennung, die nur ihm gebührt, zu holen. } \\
\text { Daraufhin lässt ihn Brean festnehmen. }\end{array}$ & $\begin{array}{l}\text { TV im Film; } \\
\text { Leitmotiv: Wahltrailer } \\
\text { Schuss - Gegenschuss - } \\
\text { Verfahren }\end{array}$ \\
\hline 36 & Begräbnis & Das Begräbnis wird im TV gezeigt. & TV im Film \\
\hline 37 & $\begin{array}{l}\text { Tod des Produzen- } \\
\text { ten und Krieg in } \\
\text { Albanien }\end{array}$ & $\begin{array}{l}\text { Eine Nachrichtensprecherin verkündet aus dem } \\
\text { Off den Tod des Produzenten Stanley Motss, } \\
\text { während man sein Haus sieht. Ein Nachrichten- } \\
\text { sprecher spricht von einem erneuten Krieg in } \\
\text { Albanien. Amerikanische Armee soll erneut } \\
\text { nach Albanien entsandt werden. } \\
\text { Als letztes Bild sieht man das Hinterzimmer im } \\
\text { Weißen Haus, in welchem die konspirativen } \\
\text { Treffen zur Erhaltung der Präsidentschaft statt- } \\
\text { fanden. }\end{array}$ & $\begin{array}{l}\text { Musik mit dem Text: } \\
\text { „Can you wag the } \\
\text { dog?“ }\end{array}$ \\
\hline 38 & Abspann & & $\begin{array}{l}\text { Musik mit dem Text: } \\
\text { „Can you wag the } \\
\text { dog?“ }\end{array}$ \\
\hline
\end{tabular}

